Der Medizinischen Fakultät der Georg-August-Universität eingereicht von Prof. Dr. med. G. Zöller

\title{
Strahlenbelastung der Augenlinse bei urologischen Eingriffen am Beispiel der ureterorenoskopischen Steinbehandlung
}

\author{
INAUGURAL-DISSERTATION \\ zur Erlangung des Doktorgrades \\ der Medizinischen Fakultät \\ der Georg-August-Universität zu Göttingen
}

vorgelegt von

Ana Sabo

aus

Novi Sad/Serbien

Göttingen 2018 
Diese Dissertation wurde 2013 bis 2017 im Klinikum Bad Hersfeld unter der Betreuung von Prof. Dr. med. G. Zöller angefertigt.

Dekan: $\quad$ Prof. Dr. rer. nat. H. K. Kroemer

Referent: $\quad$ Prof. Dr. med. G. Zöller

Ko-Referentin: $\quad$ Prof. Dr. rer. nat. B. Meller

Datum der mündlichen Prüfung: $\quad$ 25.09.2018 
Hiermit erkläre ich, die Dissertation mit dem Titel „Strahlenbelastung der Augenlinse bei urologischen Eingriffen am Beispiel der ureterorenoskopischen Steinbehandlung" eigenständig angefertigt und keine anderen als die von mir angegebenen Quellen und Hilfsmittel verwendet zu haben.

Göttingen, den 22.08.2018 Ana Sabo 


\section{Inhaltsverzeichnis}

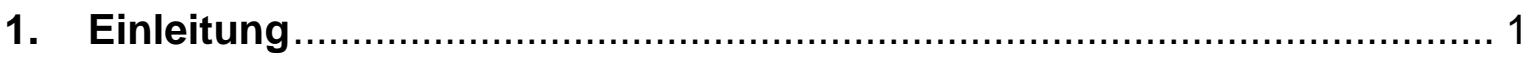

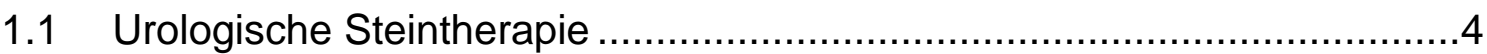

1.2 Allgemeine Strahlenbelastung und Strahlenfolgen................................14

1.3 Strahlensensibilität der Augenlinse ...............................................18

1.4 Physikalische Größen zur Dosisbestimmung .....................................20

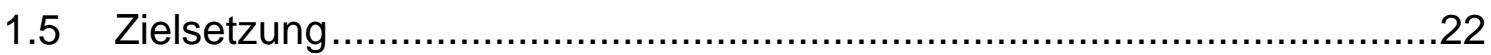

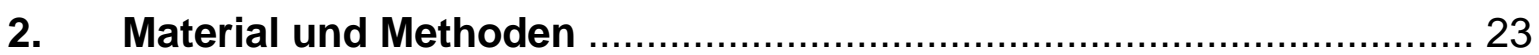

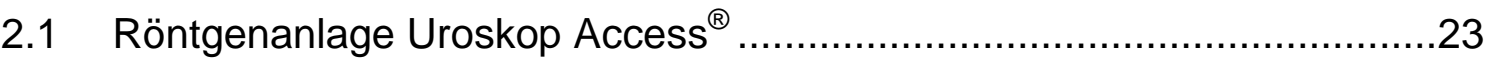

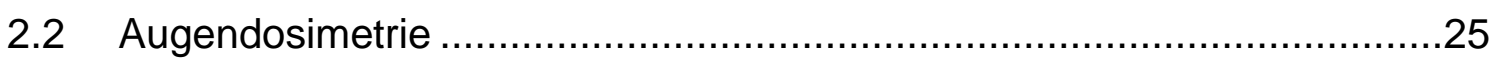

2.3 Untersuchungen am Phantommodell ..................................................26

2.4 Untersuchungen unter Realbedingungen...........................................

2.5 Datenerhebung, Datenauswertung und statistische Methoden .................31

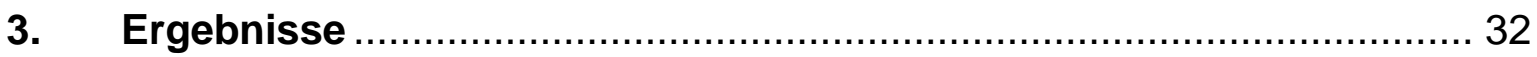

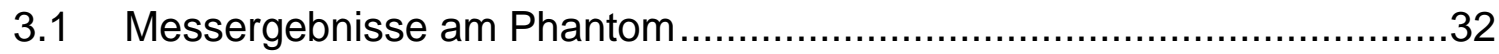

3.1.1 Messungen mit zunehmender Anzahl an Röntgenbildern ............... 32

ohne Strahlenschutzvisier .............................................................. 32

3.1.2 Messungen mit zunehmender Anzahl an Röntgenbildern ................ 32

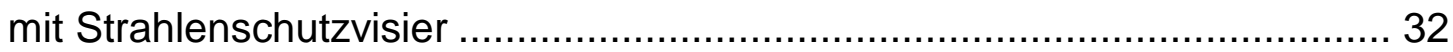

3.1.3 Messungen mit zunehmender Durchleuchtungszeit ....................... 35

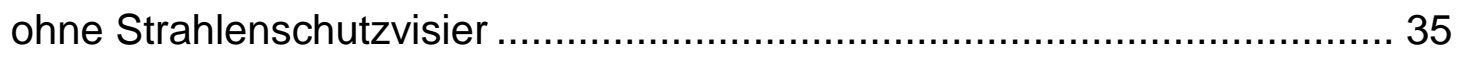

3.1.4 Messungen mit zunehmender Durchleuchtungszeit ........................ 35

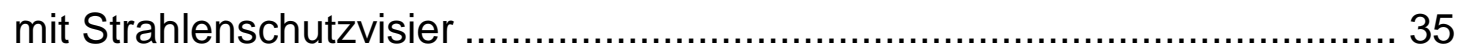

3.1.5 Vergleich der Messungen ohne und mit Strahlenschutzvisier .......... 38

3.2 Messergebnisse unter Realbedingungen ......................................... 


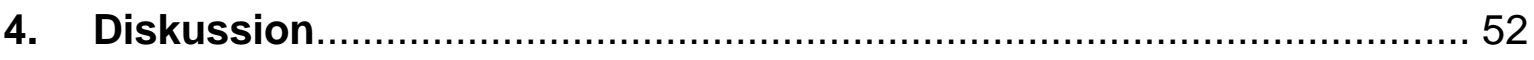

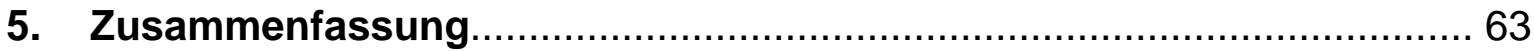

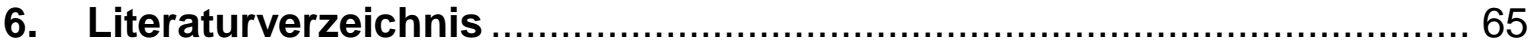




\section{Abkürzungsverzeichnis}

Charr. Charrière

CT

Computertomographie

DFP

Dosisflächenprodukt

DGU

Deutsche Gesellschaft für Urologie

DJ

Doppel-J-Schiene

DNA

deoxyribonucleic acid (DNA, Desoxyribonucleinsäure)

ESWL

extrakorporale Stoßwellenlithotripsie

Gy

Gray

Holmium-YAG-

Holmium-dotierter Yttrium-Aluminium-Granat-LASER

LASER

$\mathrm{Hp}(3)$

Personen-Äquivalentdosis in $3 \mathrm{~mm}$ Tiefe (Augenlinsenpersonendosis)

HU Hounsfield Units

J

Joule

$\mathrm{kV}$

Kilovolt

LASER

light amplification by stimulated emission of radiation

(Lichtverstärkung durch stimulierte Strahlenfreisetzung)

$\mu \mathrm{Gy}$

Mikrogray

$\mathrm{mSv}$

Millisievert

OP

Operation

$\mathrm{p}$

probability (Signifikanzwert)

PNL

perkutane Nephrolitholapaxie

$S$

Sekunden

Sv

Sievert

URS

Ureterorenoskopie 


\section{Einleitung}

Das Harnsteinleiden zählt mit einer Inzidenz von ca. 1,47 Neuerkrankungen/100 Einwohner/Jahr und einer Prävalenz von 4,7\% in der Bevölkerung zu den Volkskrankheiten in Deutschland - mit weiter steigender Tendenz (Hesse et al. 2003). Entsprechend nimmt die konservative und interventionelle Therapie von Nierenund Harnleitersteinen einen erheblichen Raum in der täglichen Arbeit in der Urologie ein. So wurden im Jahr 2016 allein am Klinikum Bad Hersfeld 316 von insgesamt 1.844 stationären Patienten $(=17,1 \%$ aller stationärer Patienten) wegen einer Urolithiasis behandelt.

Die operative Therapie der Urolithiasis war dabei in den letzten Jahrzehnten einem erheblichen Wandel unterzogen. Die klassischen offen-operativen Therapieverfahren wie die Nephro- und Ureterolithotomie wurden von minimalinvasiven Therapieverfahren wie der extrakorporalen Stoßwellenlithotripsie (ESWL), der ureterorenoskopischen Steinentfernung (URS) und der perkutanen Nephrolitholapaxie (PNL) fast vollständig verdrängt. Aufgrund der gerätetechnischen Weiterentwicklungen mit einer hohen Rate an unmittelbarer Steinfreiheit nimmt heute die ureterorenoskopische Steinentfernung zahlenmäßig die zentrale Rolle in der urologischen Steintherapie ein. So wurden am Klinikum Bad Hersfeld im Jahr 2016194 Ureterorenoskopien zur Behandlung einer Urolithiasis durchgeführt (Tab.1). Dies entspricht einem Anteil von 90,7\% aller operativen Eingriffe zur Behandlung von Nieren- oder Harnleitersteinen und 72,4\% aller durchgeführten ureterorenoskopischen Eingriffe (Tab.1 und Tab.2).

Wie auch in anderen Fachdisziplinen geht die Entwicklung minimal-invasiver endoskopischer Therapieverfahren einher mit der Notwendigkeit einer ultraschalloder radiologisch geführten intraoperativen Kontrolle. Die Strahlenbelastung für Patienten bei der minimal-invasiven Steintherapie ist in der Urologie hinreichend gut untersucht, auch durch eigene Untersuchungen am Klinikum Bad Hersfeld (Vowinkel 2011).

Untersuchungen zur Strahlenbelastung des Operateurs liegen aber kaum vor. Insbesondere fehlen aussagekräftige Untersuchungen zur Strahlenbelastung des Auges bei endourologischen Eingriffen. 
Dabei ist aus anderen Fachdisziplinen mit hoher Strahlenbelastung, wie der interventionellen Kardiologie, bekannt, dass eine hohe Strahlenbelastung der Augen mit einer hohen Inzidenz der strahlenassoziierten Katarakterkrankung des Operateurs einhergeht.

In der vorliegenden Arbeit wird deshalb der Frage der Strahlenbelastung des Auges und der daraus folgenden Strahlenbelastung der Augenlinse des Operateurs bei der ureterorenoskopischen Steinentfernung nachgegangen. 


\section{$\underline{\text { Tabelle } 1}$}

Operative Behandlung der Urolithiasis an der Klinik für Urologie und Kinderurologie am Klinikum Bad Hersfeld im Jahr 2016

\begin{tabular}{|l|l|c|}
\hline \multirow{3}{*}{ Steinlokalisation } & operativer Eingriff & Eingriffszahl \\
\hline \multirow{5}{*}{ Nierensteine } & perkutane Litholapaxie & 20 \\
\cline { 2 - 3 } & Ureterorenoskopie & 69 \\
\cline { 2 - 3 } & extrakorporale Stoßwellenlithotripsie & 0 \\
\cline { 2 - 3 } & offen-chirurgischer Eingriff & 0 \\
\hline \multirow{3}{*}{ Harnleitersteine } & Ureterorenoskopie & 125 \\
\cline { 2 - 3 } & extrakorporale Stoßwellenlithotripsie & 0 \\
\cline { 2 - 3 } & offen-chirurgischer Eingriff & 0 \\
\hline
\end{tabular}

Tabelle 2

Ureterorenoskopien an der Klinik für Urologie und Kinderurologie am Klinikum Bad Hersfeld im Jahr 2016

\begin{tabular}{|l|c|}
\hline Indikation & Eingriffszahl \\
\hline Behandlung von Nierensteinen & 69 \\
\hline Behandlung von Harnleitersteinen & 194 \\
\hline Endoskopische Histologiegewinnung im oberen Harntrakt & 18 \\
\hline Diagnostische Ureterorenoskopie ohne weitere Maßnahmen & 56 \\
\hline
\end{tabular}




\subsection{Urologische Steintherapie}

Typisches klinisches Zeichen eines symptomatischen Nieren- oder Harnleitersteines ist die Nierenkolik mit Flankenschmerzen und Schmerzausstrahlung in die entsprechenden Head'schen Zonen (Dermatom Th9-L1/2 ipsilateral). Im Urin findet sich bei $90 \%$ der Patienten eine Mikrohämaturie. Die initiale Diagnostik erfolgt durch die Ultraschalluntersuchung der Nieren, die einen Nierenstein oder die konsekutive Stauung bei einem okkludierenden Harnleiterstein darstellen kann. Die Diagnosesicherung erfolgt durch radiologische Untersuchungen, heutzutage in der Regel durch eine low-dose-nativComputertomographie des Abdomens, die einen Nieren- oder Harnleiterstein mit einer Sensitivität von $94-100 \%$ bei einer Spezifität von $92-100 \%$ beweisen kann (Leitlinie Urolithiasis 2015).

Die Therapie des Harnsteinleidens unterteilt sich in die Akuttherapie zur Beherrschung der klinischen Symptomatik und in die definitive Steintherapie mit der heute in der Regel minimal-invasiv durchgeführten Steinentfernung.

Die Akuttherapie umfasst eine suffiziente Schmerztherapie in einem Stufenkonzept mit nicht-steroidalen Antiphlogistika, Metamizol oder Morphinderivaten. Die Harnleiterobstruktion muss bei klinischen Zeichen eines Infektes (z. B. Fieber oder Leukozytose) oder bei Zeichen der Nierenfunktionseinschränkung (z. B. Kreatininerhöhung) durch die Einlage von Harnleiterschienen oder selten durch die perkutane Harnableitung mittels Nierenfistelkatheter entlastet werden. Zudem dient die Harnleiterschienung häufig der Vorbereitung einer nachfolgenden ureterorenoskopischen Steinentfernung, da die Harnleiterschiene zu einer Erweiterung des Harnleiters führt, was nachfolgende endoskopische Eingriffe erleichtert.

Für die definitive Steintherapie stehen heute unterschiedliche minimal-invasive Therapieverfahren zur Verfügung. Offen-operative Steinsanierungen erfolgen heute nur noch dann, wenn zusätzliche anatomische Veränderungen wie Ureterabangsengen operativ korrigiert werden müssen. Minimal-invasive Therapieverfahren umfassen die extrakorporale Stoßwellenlithotripsie, die perkutane Nephrolitholapaxie sowie die ureterorenoskopische Steinentfernung. 


\section{- extrakorporale Stoßwellenlithotripsie (ESWL)}

Das Prinzip der ESWL besteht darin, dass extrakorporal akustische Stoßwellen erzeugt und über einen Ankoppelbalg in den Patientenkörper eingebracht werden. Durch akustische Linsen wird dabei eine Fokussierung der Stoßwellenfront im Bereich des Nieren- oder Harnleitersteines erreicht. An den akustischen Grenzflächen zwischen Gewebe und Stein bzw. Urin und Stein entstehen durch die Impedanzsprünge starke Druck- und Zugspannungen, die auf den kristallinen Stein wirken und zu dessen Fragmentierung führen. Die entstehenden Steinfragmente müssen dann via naturalis aus dem Körper ausgeschieden werden. Je nach ESWL-Gerätesystem werden dabei die Stoßwellen durch unterschiedliche physikalische Prinzipien generiert. Die gängigen ESWL-Systeme verwenden in der Regel eine elektromagnetische, elektrohydraulische oder piezolelektrische Stoßwellengenerierung. Zur Fokussierung der akustischen Stoßwellen müssen die Nieren- oder Harnleitersteine im Körper lokalisiert werden. Dies ist durch Sonographie oder mittels Röntgensystem möglich. Moderne ESWLSysteme lassen dabei sowohl die ultraschallgeführte als auch die radiologisch geführte Steinortung zu. Für eine wirkungsvolle Steindesintegration müssen ca. 1500 bis 2000 Stoßwellen in den Körper appliziert werden. Die fortschreitende Steindesintegration lässt sich wiederum durch Sonographie oder radiologische Methoden (Röntgenaufnahme oder Durchleuchtungsbild) kontrollieren. Bei der ESWL handelt es sich um ein minimal-invasives Therapieverfahren. Die bei der Applikation der Stoßwellen an der Haut entstehenden Schmerzen lassen sich in der Regel durch eine adäquate lokale oder systemische Analgosedierung beherrschen.

Abhängig von der Steinlokalisation, der Steingröße und der Steinzusammensetzung lassen sich mit der ESWL unterschiedliche Steinfreiheitsraten erreichen. Problematisch sind Steinlage in der unteren Nierenkelchgruppe (Steinfreiheitsraten von 50 bis $60 \%$ - Leitlinie Urolithiasis 2015) und Steingrößen über $20 \mathrm{~mm}$ (Steinfreiheitsrate $75 \%$, jedoch bei $75,4 \%$ erst nach mehreren Sitzungen (Hassan et al. 2015)). 
Bei großer Steinmasse ist zudem mit dem Risiko der Harnleiterobstruktion durch die entstehenden Steinfragmente zu rechnen (Steinstraße). Hier werden dann Sekundärmaßnahmen zur Sicherung des Harnabflusses wie die Einlage von Harnleiterschienen notwendig. Bei der ESWL müssen die Steinfragmente via naturalis ausgeschieden werden. Geschieht dies nicht, so bilden die verbleibenden Restkonkremente einen Kristallisationspunkt für eine erneute Steinbildung bzw. für erneutes Steinwachstum.

\section{- ureterorenoskopische Steinentfernung}

Bei der ureterorenoskopischen Steinsanierung werden zur Steinentfernung in Steinschnittlage retrograd über die Harnröhre und die Harnblase miniaturisierte Operationsendoskope in den Harnleiter bzw. in das Nierenbeckenkelchsystem eingeführt (Abb.1 und 2). Die Ureterorenoskopie erfolgt heute in der Regel unter endoskopischer Kontrolle über Videokameras, die das endoskopische Bild auf Bildschirme übertragen (Abb.3). Zudem muss die Gerätelage im Körper jederzeit radiologisch über Röntgensysteme kontrollierbar sein. Die zusätzliche retrograde Gabe von Kontrastmitteln über die Ureterorenoskope erleichtert die radiologische Orientierung im Hohlsystem (Abb.4).

Harnleitersteine und Steine im Nierenbecken bzw. im oberen Nierenkelchbereich können dabei mittels semirigider Ureterorenoskope erreicht werden (Abb.4). Semirigide Ureterorenoskope bestehen aus Stablinsensystemen, die eine gewisse Abbiegung des Ureterorenoskops erlauben. An der Klinik für Urologie und Kinderurologie des Klinikums Bad Hersfeld werden semirigide Ureterorenoskope der Fa. Storz, Tuttlingen, Deutschland, verwendet. Für die Ureterorenoskopie werden dabei regelhaft Ureterorenoskope von $43 \mathrm{~cm}$ Länge mit einem zunehmenden Durchmesser von 9,5 Charr. an der Gerätespitze bis 13,5 Charr. an der Gerätebasis eingesetzt (Typenbezeichnung 27002 L). Diese Geräte verfügen neben dem Stablinsensystem über einen Spülkanal zur kontinuierlichen Spülung des Hohlsystems und einen separaten Arbeitskanal, über den Arbeitsgeräte (Fasszangen, Dormia-Körbchen, Lithotripsie-Systeme) in den Harnleiter eingebracht werden können (Abb.2). 
Ist der Harnleiter für das 9,5 Charr.-Ureterorenoskop nicht passierbar, so stehen zusätzlich Ureterorenoskope von $43 \mathrm{~cm}$ Länge mit einem zunehmenden Außendurchmesser von 8 Charr. an der Gerätespitze bis 12 Charr. an der Gerätebasis (Typenbezeichnung 27001 L) zur Verfügung. Allerdings ist bei diesen weiter miniaturisierten Ureterorenoskopen der Arbeitskanal nur 4 Charr. weit, was die Einsatzmöglichkeiten der Arbeitsgeräte wie Dormia-Körbchen oder Zangen im Harnleiter limitiert.

Steine in der mittleren oder unteren Nierenkelchgruppe sind mit semirigiden Ureterorenoskopen nicht erreichbar. Hier kommen flexible Ureterorenoskope mit Fiberglasoptiken zum Einsatz (Abb.4). Wir verfügen an der Klinik für Urologie und Kinderurologie des Klinikums Bad Hersfeld wiederum über Geräte der Fa. Storz, Tuttlingen, Deutschland (Typenbezeichnung Flex-X²). Die Fiberglasoptiken erlauben eine aktive Abbiegung der Endoskope an der Gerätespitze in zwei Ebenen um bis zu $270^{\circ}$ (Abb.2). Der Gerätedurchmesser beträgt 7,5 Charr. Neben der Fiberglasoptik verfügen die Geräte über einen separaten Spülkanal zur kontinuierlichen Spülung des Harntraktes sowie über einen Arbeitskanal von 3,6 Charr., über den dünne Arbeitsgeräte wie Dormia-Körbchen oder LASER-Fasern in die Nierenkelche vorgeführt werden können.

Nach endoskopischer Lokalisation des Steines können kleine Steine mittels Fasszangen oder Steinkörbchen (Dormia-Körbchen) direkt geborgen werden (Abb.3). Ist dies aufgrund der Steingröße nicht möglich, so müssen die Steine vor einer geplanten Steinbergung zunächst desintegriert werden (intrakorporale Lithotripsie). Für die intrakorporale Lithotripsie stehen unterschiedliche Gerätesysteme zur Verfügung. Bei der ballistischen Lithotripsie werden die Steine mechanisch mittels einer Metallsonde zerstört, die wie ein Presslufthammer direkt auf den Stein einwirkt. Bei der Ultraschall-Lithotripsie werden mittels Ultraschall Vibrationen erzeugt, die ebenfalls über eine Metallsonde auf den Stein übertragen werden und den Stein fragmentieren. Beide Systeme sind aufgrund des Wirkprinzips nur bei der semirigiden Ureterorenoskopie in gerade Geräten einsetzbar. 
An der Klinik für Urologie und Kinderurologie erfolgt die intrakorporale Lithotripsie deshalb mittels eines Holmium:YAG-LASER-Systems (Sphinx ${ }^{\circledR}$ Holmium:YAGLASER, Fa. LISA laser products, Katlenburg-Lindau, Deutschland). Die LASERFasern mit einem Außendurchmesser von $420 \mu \mathrm{m}$ (FlexiFib) bzw. $730 \mu \mathrm{m}$ (PercuFib, beide von der Fa. LISA laser products, Katlenburg-Lindau, Deutschland) lassen sich sowohl durch die semirigiden Ureterorenoskope als auch durch flektierte flexible Ureterorenoskope zum Stein vorführen. Der erzeugte LASERStrahl verursacht beim Auftreffen auf den Stein eine Plasmablase, die zur Fragmentierung des Steins führt (Abb.3). Die Steinfragmente können dann mittels Steinfasszangen oder Dormia-Körbchen entfernt werden.

Es handelt sich bei der ureterorenoskopischen Steinentfernung um ein minimalinvasives Therapieverfahren, das allerdings im Gegensatz zur ESWL in Vollnarkose durchgeführt wird. Vorteil der ureterorenoskopischen Steinentfernung ist, dass das Steinmaterial unmittelbar bei der Operation endoskopisch entfernt wird und die Steinfreiheit am OP-Ende endoskopisch kontrolliert werden kann. Nachteile der Ureterorenoskopie sind mögliche Verletzungen des Harnleiters durch die Ureterorenoskope selbst oder infolge der Manipulation mittels LASERLithotripsie und durch die mechanische Steinbergung sowie Anästhesieassoziierte Komplikationen (Leitlinie Urolithiasis 2015).

\section{- perkutane Nephrolitholapaxie (PNL)}

Bei größeren Steinen im Nierenbeckenkelchbereich kann durch die Ureterorenoskopie oder die ESWL keine ausreichende Steinfreiheitsrate mehr erreicht werden. Deshalb besteht hier die Indikation zur perkutanen Nephrolitholapaxie, dem invasivsten der minimal-invasiven Therapieverfahren. Hierbei wird unter sonographischer und radiologischer Kontrolle die Niere anpunktiert und ein perkutaner Operationskanal zum Nierenbecken etabliert. Nach Aufbougierung des Operationskanals können Standard-Nephroskope mit einem Außendurchmesser von 26 bis 30 Charr. perkutan in die Niere vorgeschoben werden. Durch die gerätetechnischen Weiterentwicklungen ist aber heute auch die sogenannte Mini-PNL über einen Operationstrakt von 14 - 21 Charr. möglich. 
Diese Mini-PNL stellt das Standardvorgehen in der Klinik für Urologie und Kinderurologie am Klinikum Bad Hersfeld dar. Die (Mini-)PNL muss in Vollnarkose durchgeführt werden. Traditionell erfolgt die Operation in Bauchlage, wobei heute, wie auch bei uns, zunehmend die Operation inklusive der Anpunktion der Niere in Rückenlage durchgeführt wird. Analog zur Ureterorenoskopie müssen dabei die Steine vor der Bergung in situ desintegriert werden. Auch hier kommen ballistische, ultraschallbasierte und LASER-basierte intrakorporale LithotripsieSysteme zur Anwendung. Die Steinbergung erfolgt analog mittels Steinfasszangen oder Dormia-Körbchen. Die Notwendigkeit zur perkutanen Nierenfistelung bedingt die im Vergleich zu den übrigen minimal-invasiven Therapieverfahren deutlich erhöhte Invasivität der Methode mit den Möglichkeiten der direkten Nierenschädigung, der Verletzung von Nachbarorganen (z.B. Darm, Gefäße) und mit dem insbesondere in Bauchlage erhöhten Narkoserisiko (Leitlinie Urolithiasis 2015).

Die verschiedenen Formen der minimal-invasiven Steintherapie kommen in unterschiedlichen Indikationsstellungen zum Einsatz. Die Indikationsstellung berücksichtigt dabei die Invasivität des Therapieverfahrens und die Wahrscheinlichkeit des Therapieerfolges gemessen an der $\mathrm{zu}$ erreichenden Steinfreiheitsrate. Steine bis max. $5 \mathrm{~mm}$ Größe können prinzipiell konservativ behandelt werden, da von einem komplikationslosen Steinabgang ausgegangen werden kann; die Wahrscheinlichkeit eines Spontanabgangs liegt hier bei ca. 68\% (46-85\%, Leitlinie Urolithiasis 2015). Insbesondere kleine Steine im distalen Harnleiter gehen mit einer Wahrscheinlichkeit von über $70 \%$ spontan ohne weitere Komplikationen ab. Dagegen liegt die Wahrscheinlichkeit eines komplikationslosen Spontanabgangs im mittleren Harnleiterdrittel bei ca. $45 \%$, im proximalen Harnleiter nur noch bei ca. 25\% (Leitlinie Urolithiasis 2015). Bleibt der Spontanabgang trotz suffizienter konservativer Maßnahmen aus oder kommt es zu Komplikationen wie therapierefraktären Schmerzen, Infektzeichen (Leukozytose, Fieber) oder Zeichen der Nierenfunktionseinschränkung (Kreatininerhöhung), so wird eine operative Steinsanierung erforderlich. 
Die aktuellen Empfehlungen der S2k-Leitlinie zum Einsatz der unterschiedlichen minimal-invasiven Therapieverfahren (ESWL, Ureterorenoskopie und perkutane Litholapaxie) in Abhängigkeit von Steinlokalisation (Nierenbecken, Nierenkelch, proximaler und mittlerer Harnleiter bzw. distaler Harnleiter) und Steingröße $(<10$ mm, $10-20$ mm bzw. > $20 \mathrm{~mm}$ ) sind in der Tabelle 3 dargestellt.

Negative Erfolgsprädikatoren für eine erfolgreiche ESWL-Behandlung sind dabei ein steiler Winkel zwischen unterem Kelch und Nierenbecken, lange untere Kelchhälse sowie die voraussichtliche Steinhärte. Hounsfield-Einheiten über 1000 HU im Nativ-CT sprechen dabei für sehr harte Steine wie Brushit, Zystin oder Calciumoxalatmonohydratsteine, die nur schwer mittels ESWL desintegriert werden können. Zudem wird die Therapieeffektivität der ESWL durch eine Adipositas sowie durch Skelettdeformitäten mit Problemen bei der Geräteankopplung beeinträchtigt (Leitlinie Urolithiasis 2015).

Wenn auch in der S2k-Leitlinie ESWL und URS bei vielen Indikationen als gleichwertig dargestellt sind, so tritt doch in den letzten Jahren durch die Weiterentwicklung der Ureterorenoskopie mit Miniaturisierung des Gerätedurchmessers und durch die Weiterentwicklung der intrakorporalen Lithotripsie mittels unterschiedlicher LASER-Systeme die ureterorenoskopische Steinentfernung immer mehr in den Vordergrund, da hier sehr häufig eine sofortige postoperative Steinfreiheit erreicht werden kann. Im Gegensatz dazu wird bei der ESWL der Stein von außen im Hohlsystem zunächst nur lithotripsiert, und die entstandenen Fragmente müssen spontan ausgeschieden werden. Dies ist ein länger dauernder Prozess. Zudem ist nicht gewährleistet, dass eine komplette Steinfreiheit erreicht wird. Das Problem der Residualsteine nach ESWL ist bekannt, ebenso die Tatsache, dass Residualsteine den Kristallisationspunkt für eine erneute Steinbildung (Rezidiv-Steinbildung) darstellen können (Leitlinie Urolithiasis 2015). Wir führen deshalb in unserer Klinik seit ca. 5 Jahren keine ESWL-Behandlungen mehr durch. Primäres operatives Verfahren zur Steinentfernung ist in unserer Klinik für alle Harnleitersteine und kleinere Nierenkelch- und Nierenbeckensteine bis $2 \mathrm{~cm}$ Größe die ureterorenoskopische Steinentfernung mit und ohne intrakorporale LASER-Lithotripsie, für Nierensteine $>2 \mathrm{~cm}$ Größe die miniaturisierte perkutane Nephrolitholapaxie (Mini-PNL). 
Während die ESWL unter alleiniger sonographischer Steinortung durchgeführt werden kann, ist bei der Ureterorenoskopie und der perkutanen Nephrolitholapaxie die intraoperative radiologische Kontrolle der Gerätelage zwingend erforderlich. Dies führt, anders als bei der rein sonographiegeführten ESWL, zu einer Strahlenexposition von Patienten, Operateur und Assistenzpersonal. Entsprechend sind persönliche Strahlenschutzmaßnahmen wie Röntgenschürzen und Schilddrüsenschutz für Operateur und Assistenzpersonal unabdingbar.

\section{Tabelle 3}

Urologische Steintherapie gemäß S2k-Leitlinie der Deutschen Gesellschaft für Urologie (Leitlinie Urolithiasis 2015)

\begin{tabular}{|l|l|l|l|}
\hline & $\begin{array}{l}\text { Kleiner Stein: } \\
\text { Harnleiter bis 10 } \\
\text { mm Niere bis 9 mm }\end{array}$ & $\begin{array}{l}\text { Mittlerer Stein: } \\
\text { Harnleiter }>10 \mathrm{~mm} \\
\text { Niere 10-20 mm }\end{array}$ & Giere $>20 \mathrm{~mm}$ \\
\hline $\begin{array}{l}\text { Nierenbecken } \\
\text { obere/mittlere } \\
\text { Nierenkelche }\end{array}$ & $\begin{array}{l}\text { 1. Wahl ESWL/URS } \\
\text { 2. Wahl PNL }\end{array}$ & $\begin{array}{l}\text { ESWL/URS/PNL } \\
\text { gleichwertig }\end{array}$ & $\begin{array}{l}\text { 1. Wahl PNL } \\
\text { 2. Wahl ESWL/PNL }\end{array}$ \\
\hline $\begin{array}{l}\text { untere Kelch- } \\
\text { gruppe ohne } \\
\text { negative Er- } \\
\text { folgsprädiktoren } \\
\text { für ESWL }\end{array}$ & $\begin{array}{l}\text { 1. Wahl ESWL/URS } \\
\text { 2. Wahl PNL }\end{array}$ & $\begin{array}{l}\text { ESWL/URS/PNL } \\
\text { gleichwertig }\end{array}$ & $\begin{array}{l}\text { 1. Wahl PNL } \\
\text { 2. Wahl ESWL/URS }\end{array}$ \\
\hline $\begin{array}{l}\text { untere Kelch- } \\
\text { gruppe mit } \\
\text { negativen Er- } \\
\text { folgsprädiktoren } \\
\text { für ESWL }\end{array}$ & $\begin{array}{l}\text { 1. Wahl ESWL/URS } \\
\text { 2. Wahl PNL }\end{array}$ & $\begin{array}{l}\text { 1. Wahl URS/PNL } \\
\text { 2. Wahl ESWL }\end{array}$ & $\begin{array}{l}\text { 1. Wahl PNL } \\
\text { 2. Wahl ESWL/URS }\end{array}$ \\
\hline $\begin{array}{l}\text { proximaler } \\
\text { Harnleiter }\end{array}$ & $\begin{array}{l}\text { 1. Wahl ESWL } \\
\text { 2. Wahl URS }\end{array}$ & $\begin{array}{l}\text { URS / ESWL } \\
\text { gleichwertig }\end{array}$ & \\
\hline $\begin{array}{l}\text { mittlerer/distaler } \\
\text { Harnleiter }\end{array}$ & $\begin{array}{l}\text { 1. Wahl URS } \\
\text { 2. Wahl ESWL }\end{array}$ & $\begin{array}{l}\text { 1. Wahl URS } \\
\text { 2. Wahl ESWL }\end{array}$ & \\
\hline
\end{tabular}




\section{Abbildung 1}

Räumliche Anordnung von Operateur, Operationstisch, Patient und Röntgenröhre (Uroskop Access ${ }^{\circledR}$, Fa. Siemens, Erlangen, Deutschland) bei der ureterorenoskopischen Steinentfernung

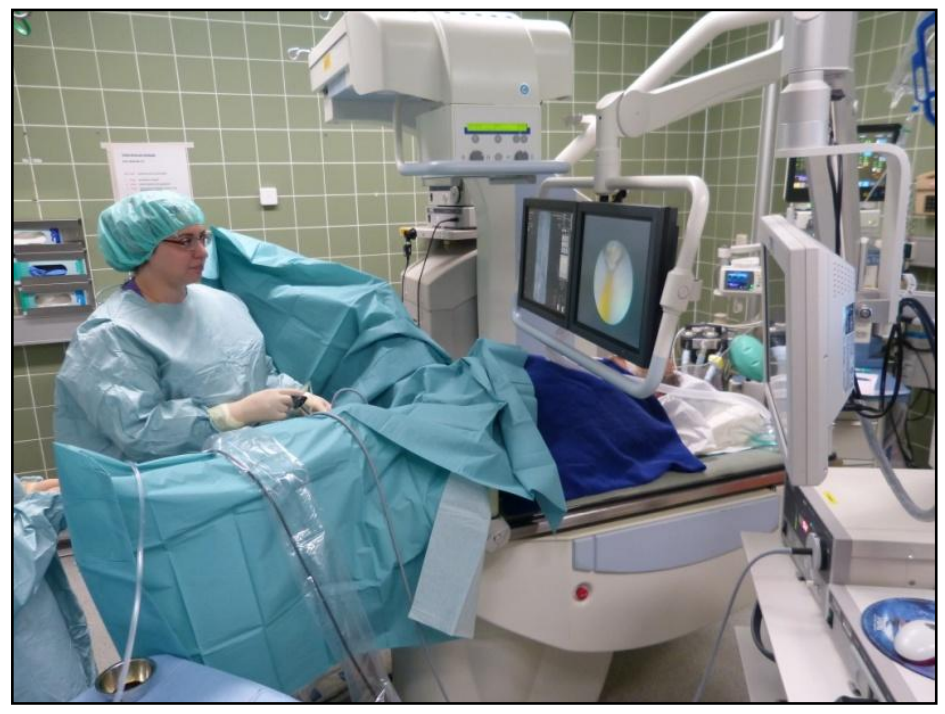

\section{Abbildung 2}

Instrumente für die ureterorenoskopische Steinentfernung: semirigides Ureterorenoskop 27002 K bzw. flexibles Ureterorenoskop Flex- $X^{2}$ (jeweils Fa. Storz, Tuttlingen), Dormia-Körbchen und Steinfasszange sowie Ex-situ-Bild einer URSSpitze mit Dormia-Körbchen und gefasstem kleinem Konkrement
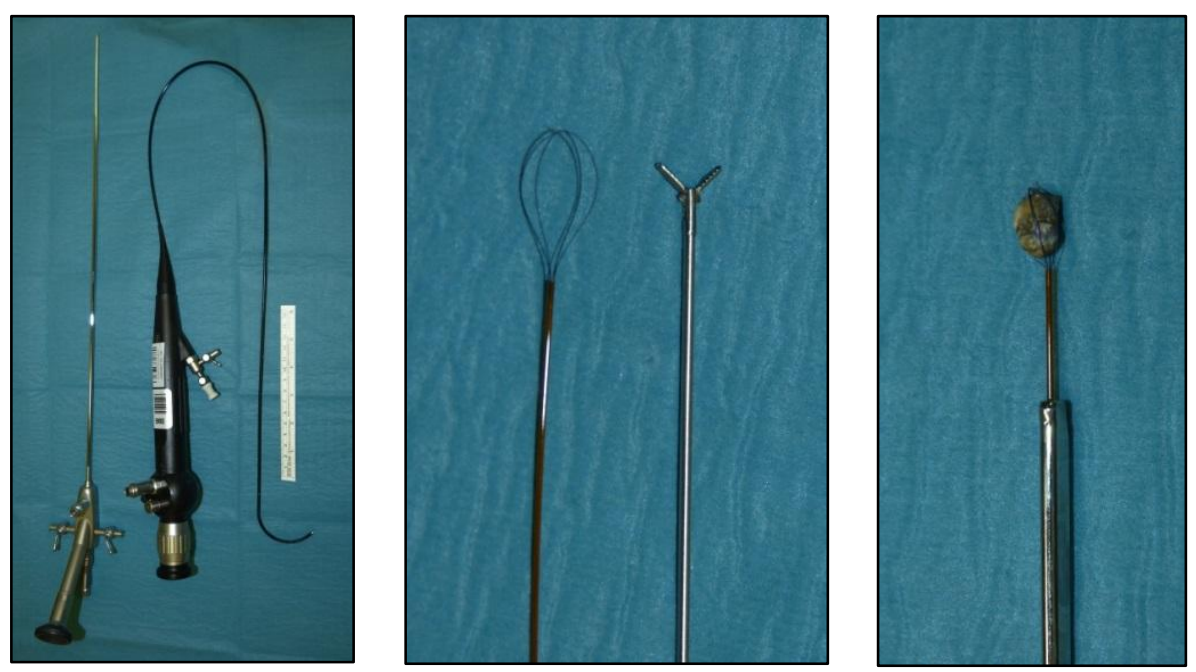


\section{Abbildung 3}

Intraoperatives endoskopisches Bild mit Steinentfernung mittels Dormia-Körbchen bzw. LASER-Lithotripsie mittels Holmium:YAG-LASER
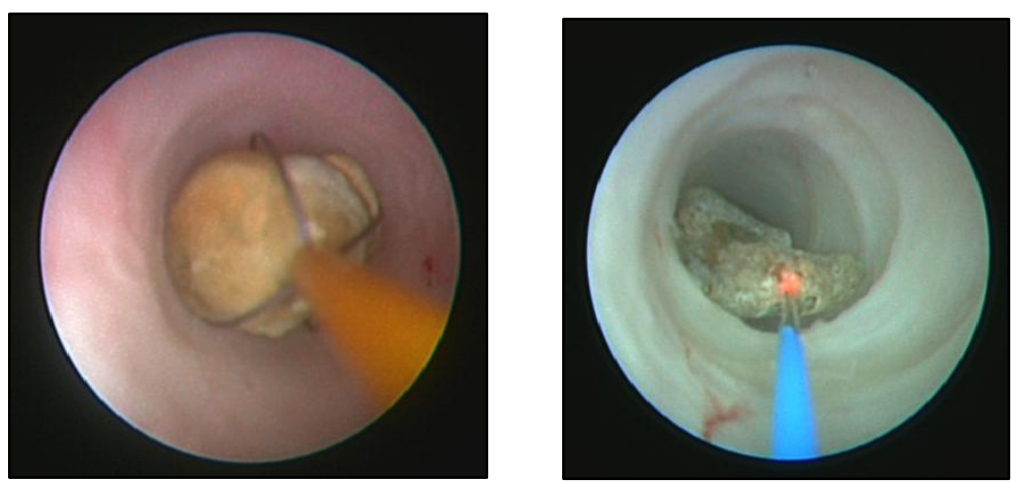

\section{Abbildung 4}

Intraoperatives Röntgenbild bei der semirigiden bzw. flexiblen Ureterorenoskopie
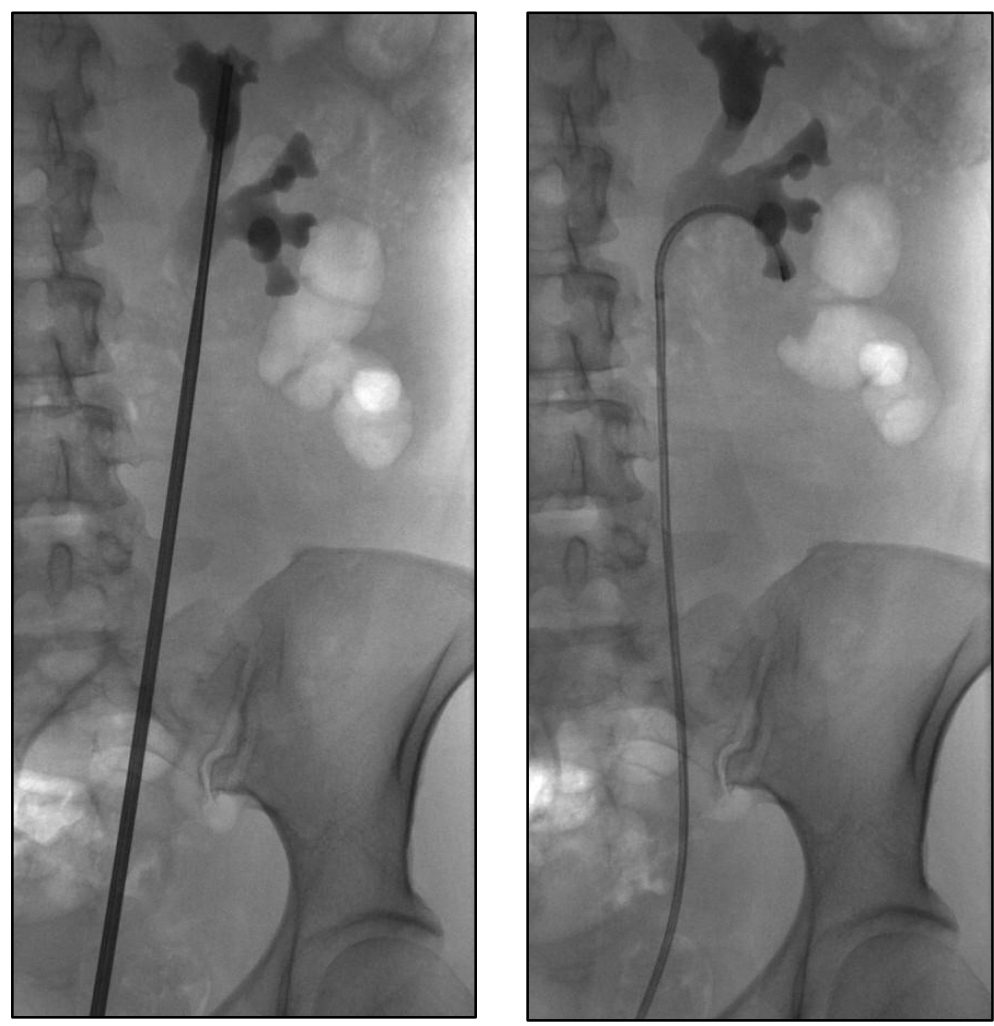


\subsection{Allgemeine Strahlenbelastung und Strahlenfolgen}

Jeder Mensch ist im Laufe seines Lebens unterschiedlichen Strahlenbelastungen ausgesetzt, wobei zwischen einer Strahlenbelastung durch natürliche bzw. durch zivilisatorisch bedingte Strahlung unterschieden wird (Bundesamt für Strahlenschutz, Stand 07/2016, 2. und 3. Internetquelle).

\section{- natürlich bedingte Strahlung}

Die natürliche Strahlenbelastung resultiert zum einen aus radioaktiven Substanzen, die in unterschiedlicher Konzentration sowohl im Erdboden wie auch in der Luft und im Wasser vorkommen (terrestrische Strahlung). Zudem sind wir einer kosmischen Strahlenbelastung ausgesetzt. Die Höhe der natürlichen Strahlenbelastung weist dabei regional starke Schwankungen auf.

\section{- zivilisatorisch bedingte Strahlung}

Zu den zivilisatorischen Strahlenbelastungen zählen beispielsweise in Kernkraftwerken erzeugte Strahlungen, die insbesondere bei Reaktorunfällen eine massive Strahlenbelastung der Bevölkerung zur Folge haben können. Die größte zivilisatorische Strahlenbelastung stellen allerdings in der Regel medizinische Untersuchungen (Röntgenuntersuchungen, nuklearmedizinische Diagnostik) und medizinische Therapieverfahren (z. B. Bestrahlungsverfahren in der Onkologie) dar. Diese Verfahren führen zur Strahlenbelastung des untersuchten bzw. behandelten Patienten, aber auch zu einer Strahlenbelastung des an der Untersuchung bzw. am Eingriff beteiligten medizinischen Personals. Dies trifft insbesondere für Operateure bei interventionellen, röntgenologisch kontrollierten Eingriffen zu. Hierbei befinden sich die Operateure zwar nicht im direkten Strahlengang, sind aber der durch den Patienten verursachten Streustrahlung ausgesetzt. Trotz persönlicher Schutzmaßnahmen wie Röntgenschürzen oder Schilddrüsenschutz kann sich hier durch die häufig und oft über Jahrzehnte durchgeführten Eingriffe eine signifikante kumulierte Strahlendosis mit dem Risiko konsekutiver Strahlenschäden ergeben. 
Strahlenschäden entstehen, wenn ionisierende Strahlung auf biologisches Gewebe trifft. Es kommt hierbei zu einer Reihe von physikalisch-chemischen Vorgängen, die über eine Kettenreaktion zu Veränderungen des Gewebes insbesondere auf DNA-Ebene führen. Daraus entstehende Mutationen können Erkrankungen oder den Tod des gesamten Organismus zur Folge haben. Die intrazellulären Prozesse bei Strahlenschädigung lassen sich in vier Stadien einteilen (Thiessen, 1978).

Das physikalische, das physikalisch-chemische und das chemische Stadium werden in Sekundenbruchteilen durchlaufen. Die energiegeladenen Teilchen der ionisierenden Strahlung treffen auf Atome bzw. Moleküle des biologischen Gewebes, wodurch es zur Übertragung von Energie auf die Moleküle des Gewebes kommt (physikalisches Stadium). Die dadurch angeregten bzw. ionisierten Atome/Moleküle reagieren daraufhin mit anderen Molekülen in ihrer Umgebung, infolge dessen sich die Struktur dieser Moleküle bis hin zum Zerfall verändern kann. Dabei können als Zerfallsprodukte freie Radikale (Molekülbruchstücke mit einem ungepaarten Elektron) entstehen (physikalisch-chemisches Stadium). Diese verursachen Sekundärreaktionen mit Molekülen in ihrer Nachbarschaft (chemisches Stadium).

Das vierte, biologische, Stadium ist wesentlich langwieriger und kann sich über Jahre oder Jahrzehnte erstrecken. Durch die Einwirkung der freien Radikale können Mutationen bzw. Schäden an der DNA auftreten, die den Stoffwechsel der Zelle verändern und beispielsweise zu unkontrolliertem Wachstum oder zum Tod der Zelle führen. Sind die Reparaturmechanismen des Organismus nicht mehr in der Lage, diese entstandenen Schäden in ausreichendem Maße zu korrigieren, manifestieren sich strahlenbedingte Krankheiten, die letztlich auch zum Tod des Organismus führen können. Dabei sind nicht alle Gewebe gleichermaßen strahlenempfindlich. Am empfindlichsten sind Zellen während ihrer Zellteilungsphase. Dementsprechend sind die strahlensensibelsten Gewebe diejenigen, deren Zellen sich häufig teilen und lange Teilungsphasen haben, sowie Zellen, die wenig differenziert sind. Dies trifft in besonderem Maße auf Tumorzellen zu, sodass diese Wirkungen in der Radioonkologie zur Bestrahlung von Tumoren genutzt werden können. 
Jedoch gibt es auch im gesunden menschlichen Organismus Gewebe, die sehr empfindlich auf Bestrahlung reagieren. Dies sind insbesondere embryonale Gewebe und die für die Reproduktion zuständigen Keimzellen, aber auch das blutbildende Knochenmark, Darmepithel- und Hautzellen sowie die Augenlinse. Die Schwere der Schädigung ist nicht zwingend dosisabhängig. Man unterscheidet hier prinzipiell zwei Wirkmechanismen, die als „deterministische“ und „stochastische“ Wirkung bezeichnet werden (Bundesamt für Strahlenschutz, Stand 05/2016).

\section{- deterministische Strahlenwirkung}

Bei der deterministischen Strahlenwirkung führt eine Dosissteigerung über eine zunehmende Zahl geschädigter Zellen zu einer zunehmenden Schwere der Strahlenschädigung. Da Organe einerseits auch mit einer gewissen Anzahl fehlerhafter Zellen noch funktionieren und andererseits auch Reparaturmechanismen eine Regeneration von Zellen bewirken können, ergibt sich für die deterministische Wirkung ein Dosisschwellenwert. Erst bei dessen Überschreitung und damit bei einer bestimmten quantitativen Schädigung des biologischen Gewebes wird die Organfunktion erkennbar beeinträchtigt und eine Strahlenschädigung manifest. Mit zunehmender Überschreitung des Grenzwerts erhöht sich der Schweregrad der Strahlenschädigung. Dies gilt beispielsweise für die strahlenbedingte Trübung der Augenlinse (Strahlenkatarakt) oder die sogenannte Strahlenkrankheit, die nach einer massiven Ganzkörperbestrahlung auftritt und oft tödlich verläuft.

Durch Strahlenschutzmaßnahmen mit einer konsekutiven Reduktion der Strahlenbelastung lassen sich deterministische Strahlenschäden reduzieren oder vermeiden. Dies trifft auch für Operateure im Rahmen radiologisch kontrollierter minimal-invasiver Therapiemaßnahmen zu.

\section{- stochastische Strahlenwirkung}

Im Gegensatz zur deterministischen Strahlenwirkung ist die stochastische Strahlenwirkung nur in geringem Maße dosisabhängig und letztlich nicht vorhersehbar. 
Sie beruht auf einer Schädigung der DNA; dabei führt die DNA-Schädigung nicht zum Zelltod sondern zu einer Mutation. Wird diese veränderte Zelle von der körpereigenen Immunabwehr nicht als krankhaft erkannt und beseitigt, so kann sie sich vermehren und infolge dessen zu einer Erkrankung führen. Über eine Schädigung des Erbguts kann auch die nachfolgende Generation von Erkrankungen oder Missbildungen betroffen sein. Die Wahrscheinlichkeit für eine durch eine stochastische Strahlenwirkung ausgelöste Erkrankung (beispielsweise Leukämie) steigt zwar mit zunehmender Strahlendosis, aber weder ist das Auftreten der Erkrankung vorhersehbar noch korreliert die Schwere der Erkrankung mit der Strahlendosis.

Da stochastische Strahlenfolgen dosisunabhängig sind und keinen Schwellenwert haben, können sie auch durch Strahlenschutzmaßnahmen nicht sicher vermieden werden. Dies ist auch für den interventionell tätigen Operateur von Bedeutung. 


\subsection{Strahlensensibilität der Augenlinse}

Die bikonvexe Augenlinse dient dazu, das durch die Pupille einfallende Licht zu bündeln, sodass auf der Netzhaut ein scharfes Bild entsteht. Die Linse besteht aus Linsenkern und Linsenrinde; diese sind von einer Kapsel umgeben und über Zonulafasern an der Aderhaut aufgehängt. Das Linsengewebe ist normalerweise transparent, Blutgefäße finden sich nicht darin. Die Nährstoffversorgung erfolgt über Diffusion aus dem Kammerwasser.

Bei der Katarakt (oder Grauen Star) kommt es zu einer Eintrübung der Augenlinse, wodurch deren Funktionalität nicht mehr gewährleistet ist - die Sehschärfe nimmt ab und das Sehvermögen schwindet; es kann letztlich zur vollständigen Erblindung kommen. Dies kann mit konservativen Methoden nicht mehr behoben werden, eine Operation ist erforderlich. Die getrübte Linse muss entfernt und eine Kunststofflinse eingesetzt werden. Je nach Lokalisation unterscheidet man nukleäre Katarakte (Kerntrübung), kortikale Katarakte (Rindentrübung, anterior oder posterior), subkapsuläre Katarakte (Trübung an der Grenze zur Linsenrinde, anterior oder posterior) oder totale Katarakte (Hahn 2012).

Neben der ionisierenden Strahlung wurden als Risikofaktoren bislang auch Infrarotstrahlung und UV-Strahlung, aber auch Alter, weibliches Geschlecht, erbliche Vorbelastung, verschiedene systemische Erkrankungen (z.B. Diabetes mellitus), Nikotinabusus, Steroidhormone, lokale Traumata und chronische Aderhautentzündungen identifiziert (Hahn 2012).

Tierexeperimentelle Studien zeigten, dass eine hohe Strahlenbelastung zu Veränderungen der DNS und einer gestörten Zellteilung führte und eine Katarakt in der posterioren subkapsulären Region auslöste (Worgul et al. 1989, Wolf et al. 2008, Zintz und Beebe 1986). Allerdings wurde hierbei eine sehr hohe Strahlendosis appliziert, in fast allen Studien mindestens 1 Gy. Die Studien wurden hauptsächlich an Mäusen und Ratten durchgeführt; andere Arbeiten konnten jedoch zeigen, dass Linsen von Nagetieren und Primaten vergleichbar aufgebaut sind, es zeigten sich keine morphologischen, physiologischen oder biochemischen Unterschiede (Malmström und Kröger 2006). 
Auch beim Menschen wurden posteriore subkapsuläre Veränderungen und kortikale Katarakte nach einer Strahlenexposition beschrieben (Worgul et al. 1989 und 1996, Nefzger et al. 1969, Ferrufino-Ponce und Henderson 2006, Nakashima et al. 2006). Die Daten entstammen größtenteils retrospektiven Beobachtungen an Überlebenden von Atomwaffenexplosionen und Reaktorunfällen, an beruflich strahlenexponierten Personen (Piloten, Astronauten, radiologisch-technische Assistenten) sowie Patienten nach medizinischer Strahlenexposition.

Die in diesem Zusammenhang interessanteste prospektive Studie wurde in den USA an über 37000 radiologisch-technischen Assistenten durchgeführt (Chodick et al. 2008); dabei wurde nachgewiesen, dass das zusätzliche relative Risiko für die Entwicklung einer Katarakt bei 2,0 pro Gy lag. Eine Schwellendosis konnte dabei nicht nachgewiesen werden. Es zeigte sich aber eine deutliche Abhängigkeit vom Alter bei Beginn der Exposition: 40- bis 44-Jährige haben im Vergleich zu $<30$-Jährigen ein 7,6-fach erhöhtes relatives Risiko.

Laut derzeitiger Strahlenschutzverordnung darf für die Exposition der Augenlinse ein jährlicher Grenzwert von 0,15 Gy (Augenlinsendosis) nicht überschritten werden (Bundesamt für Strahlenschutz, Stand 07/2016, 1. Internetquelle). Bei einer 20-30 Jahre dauernden beruflichen Exposition würde dies einer Kumulativdosis von 3-4,5 Gy entsprechen. 


\subsection{Physikalische Größen zur Dosisbestimmung}

Um die Strahlenwirkung auf biologische Gewebe zu quantifizieren, verwendet man unterschiedliche Begriffe bzw. physikalische Größen (Shannoun et al. 2009):

\section{- Energiedosis}

Die in Gray gemessene Energiedosis gibt an, wie viel Strahlenenergie ein kg Materie absorbiert $(\mathrm{Gy}=\mathrm{J} / \mathrm{kg}$ ).

\section{- Äquivalentdosis}

$\mathrm{Da}$ unterschiedliche Strahlungsarten eine unterschiedliche biologische Wirksamkeit aufweisen, wird zur genaueren Differenzierung die Äquivalentdosis berechnet. Diese entspricht der Energiedosis multipliziert mit einem Strahlenwichtungsfaktor und wird in Sievert ausgedrückt $(1 \mathrm{~Sv}=\mathrm{J} / \mathrm{kg})$. Der Wichtungsfaktor beträgt für die medizinisch relevante Röntgen- und Gammastrahlung 1, sodass hier die Äquivalentdosis der Energiedosis entspricht. Für andere Strahlungsarten (z. B. Alphateilchen, Neutronen) kann der Wichtungsfaktor bis 20 betragen.

\section{- Organdosis und Augenlinsenpersonendosis}

Als Organdosis bzw. Organ-Äquivalentdosis wird die in einem Organ, Gewebe oder Körperteil aufgenommene und mit dem Strahlenwichtungsfaktor multiplizierte Energiedosis bezeichnet; sie wird in Sv ausgedrückt.

Die Augenlinsenpersonendosis $\mathrm{Hp} \mathrm{(3)} \mathrm{ist} \mathrm{eine} \mathrm{Organ-Äquivalentdosis,} \mathrm{die} \mathrm{in} 3 \mathrm{~mm}$ Tiefe (entsprechend der Lage der Augenlinse) bestimmt und in Sv angegeben wird. Dabei wird am Körper einer im Strahlenfeld befindlichen Person gemessen.

- effektive Dosis

Da der Effekt der Strahlung nicht nur von der Art der Strahlung, sondern auch von der Art des bestrahlten Gewebes abhängt, wurde der Begriff "effektive Dosis“ eingeführt. Die effektive Dosis ist die Summe der mit einem Gewebewichtungsfaktor multiplizierten Äquivalentdosen der Körperorgane und Körpergewebe. Dabei bildet der Gewebewichtungsfaktor die Strahlensensitivität des Gewebes ab: so hat z. B. die vergleichsweise strahlenunempfindliche Haut einen Gewebewichtungsfaktor von 0,01, während die strahlenempfindlichen 
Keimdrüsen (Eierstöcke, Hoden) einen deutlich höheren Gewebewichtungsfaktor aufweisen. Die effektive Dosis wird ebenso wie die Äquivalentdosis in Sv angegeben.

\section{- Dosisflächenprodukt}

In der medizinischen Praxis ist die effektive Dosis kein geeignetes Mittel zur Quantifizierung der Strahlenbelastung, da sie nicht direkt gemessen werden kann. Stattdessen hat sich hierfür das sogenannte Dosisflächenprodukt durchgesetzt.

Dabei wird durch eine Multiplikation der Energiedosis mit der bestrahlten Fläche $\left(G y^{*} \mathrm{~cm}^{2}\right.$ bzw. $\left.\mu G y^{*} \mathrm{~m}^{2}\right)$ ein gut verfügbarer und überprüfbarer Näherungswert ermittelt. Dies geschieht über eine direkt im Strahlengang platzierte Ionisationskammer. Die während der Untersuchung automatisch berechneten Werte werden vom Operateur aufgerufen und im erstellten Röntgenbericht dokumentiert.

Zur Berechnung der effektiven Dosis kann das Dosisflächenprodukt mit einem untersuchungsspezifischen Konversionsfaktor multipliziert werden. 


\section{$1.5 \quad$ Zielsetzung}

In der vorliegenden Arbeit wurde untersucht, wie hoch die Strahlenbelastung des Auges des Operateurs bei der ureterorenoskopischen Steinentfernung ist und ob durch die Verwendung von Strahlenschutzvisieren eine signifikante Senkung der Strahlenbelastung am Auge erreicht werden kann.

Die Untersuchungen erfolgten zum einen unter standardisierten Versuchsbedingungen im Untersuchungsmodell (Alderson-Phantom und Kopfphantom), zum anderen aber auch unter realen Operationsbedingungen bei ureterorenokopischen Steinentfernungen von Nieren- oder Harnleitersteinen bei Patienten des Klinikums Bad Hersfeld.

Alle Untersuchungen bzw. operativen Eingriffe wurden von der Promovendin durchgeführt, um interindividuelle Unterschiede zwischen den Operateuren als Fehlerquelle auszuschließen. 


\section{Material und Methoden}

\subsection{Röntgenanlage Uroskop Access ${ }^{\circledR}$}

Ureterorenoskopische Eingriffe werden unter Röntgenkontrolle durchgeführt. Hierfür steht an der Klinik für Urologie und Kinderurologie Bad Hersfeld die Röntgeneinheit Uroskop Access ${ }^{\circledR}$ der Fa. Siemens, Erlangen, Deutschland, als Röntgenarbeitsplatz zur Verfügung. Das Uroskop Access ${ }^{\circledR}$ besteht aus dem Obertischröntgengenerator POLYDOROS SX 65/80 mit Belichtungsautomatik und aus einer Patientenlagerungsplatte. Diese Lagerungsplatte erlaubt eine adäquate Patientenlagerung (z. B. Steinschnittlagerung bei der Ureterorenoskopie) bei freiem Zugang zum Patienten für Anästhesie, Operateur und Assistenzpersonal (Abb.5). Das Röntgengerät und die motorgetriebene Tischbewegung können zum einen über eine Bedienkonsole am Röntgengerät selbst sowie über eine Bedienkonsole am Arztarbeitsplatz bedient werden. Die intraoperative Bedienung durch den Operateur erfolgt über einen Multifunktionsfußschalter.

Für die unterschiedlichen diagnostischen und therapeutischen Verfahren in der Urologie sind dabei in der Röntgeneinheit Uroskop Access ${ }^{\circledR}$ spezifische Organprogramme für die Durchleuchtung oder für die Röntgenaufnahmen vorprogrammiert. Die Ureterorenoskopie erfolgt dabei an unserer Klinik unter Verwendung des Organprogramms „Retrograde Pyelographie“ mit einer Röhrenspannung von $79 \mathrm{kV}$, mit einem Röhren-Fokus-Abstand von $115 \mathrm{~cm}$, Streustrahlenraster von 17:1 und mit einem Kupferfilter von 0,2 mm für die Durchleuchtung und 0,1 mm für Röntgenaufnahmen. Das Bild wird situationsgerecht mit Irisblenden eingeblendet.

Die Ureterorenoskopie erfolgt unter endoskopischer Sicht mittels Videokette, jedoch mit regelmäßiger Bildwandlerkontrolle (Steinlokalisation, Darstellung der ableitenden Harnwege) und intraoperativer Dokumentation mittels Röntgenaufnahmen oder Last-image-hold-Aufnahmen (Speicherung des letzten Durchleuchtungsbildes ohne zusätzliche Strahlenbelastung). 
Das endoskopische Bild wie auch die Röntgenaufnahmen werden auf zwei Bildschirmen an der Röntgenanlage Uroskop Access ${ }^{\circledR}$ wiedergegeben, sodass der operative Eingriff anhand dieser Videomonitore fortwährend kontrolliert werden kann.

Die Messung der Strahlendosis erfolgt als Messung des Dosisflächenprodukts (wiedergegeben in $\mu \mathrm{Gy}^{*} \mathrm{~m}^{2}$ ) mittels einer direkt in den Strahlengang eingebrachten Dosismesskammer. Dosisflächenprodukt, Röhrenspannung, Durchleuchtungszeit und Anzahl der Röntgenbilder bzw. der abgespeicherten Last-image-hold-Bilder werden im System dokumentiert.

\section{Abbildung 5}

Röntgenanlage Uroskop Access ${ }^{\circledR}$

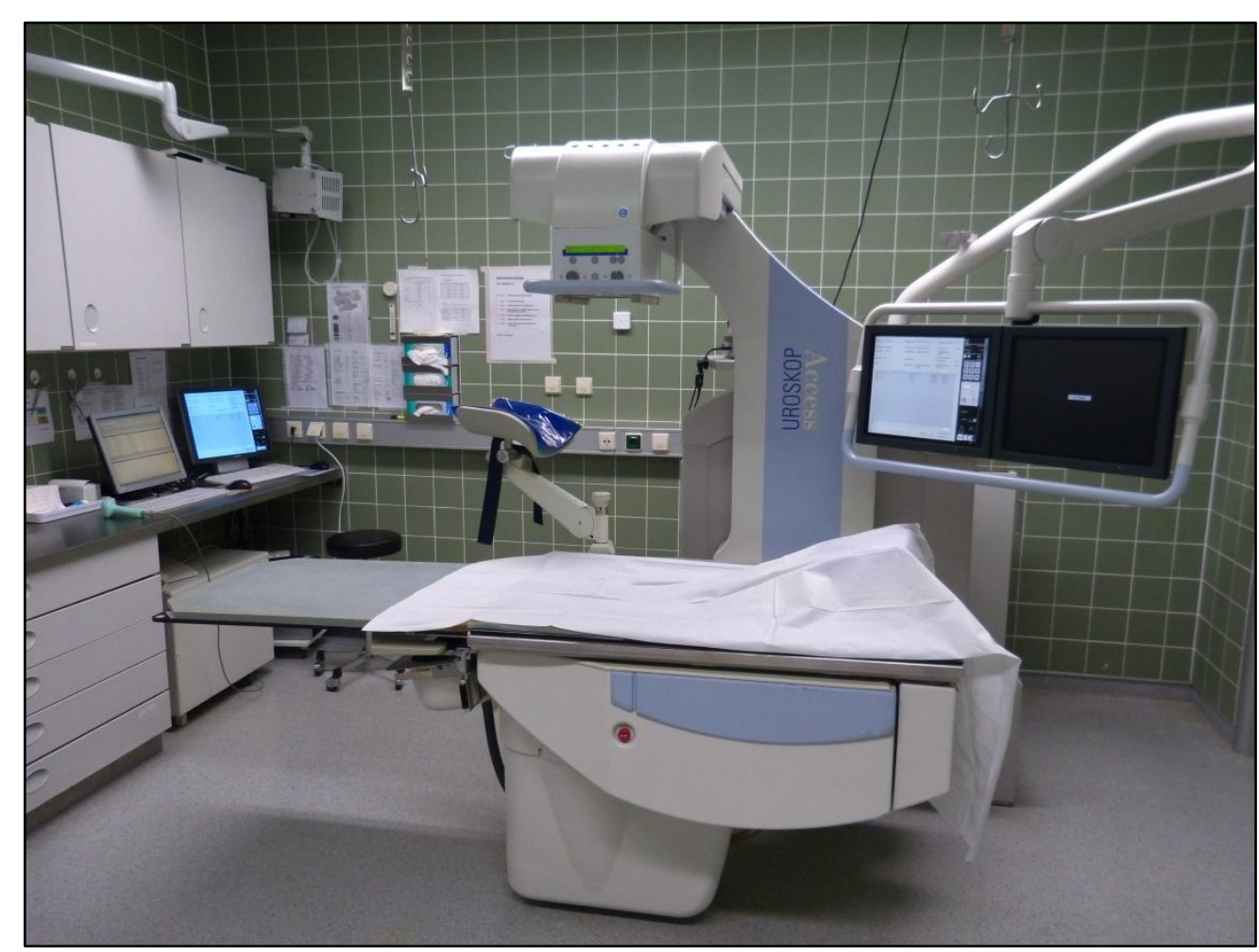




\subsection{Augendosimetrie}

Die Augendosimetrie erfolgte mittels des Thermolumineszenz-Augenlinsendosimeters Eye- $D^{\circledast}$ der Fa. Radcard, Polen. Diese Augendosimeter wurden uns von der Auswertungsstelle für Strahlendosimeter des Helmholtz Zentrums München zur Verfügung gestellt und auch dort ausgewertet. Als Messgröße wurde die Teilkörperdosismessgröße für die Augenlinsendosis $\mathrm{Hp}(3)$ verwendet.

Um ermitteln zu können, welche Dosis ausschließlich durch die untersuchungsbedingte Streustrahlung entsteht, musste der Effekt der natürlichen Umgebungsstrahlung herausgerechnet werden, welcher beim Transport und bei Lagerung der Augendosimeter zu einer zusätzlichen Strahlenbelastung der Augendosimeter führt. Hierfür wurden bei jedem Untersuchungsdurchgang 6 Augendosimeter nicht für die Untersuchungen verwendet, sondern ohne weitere Belichtung ausgewertet. Aus den Messwerten wurde anschließend der arithmetische Mittelwert gebildet (Nullwert). Dieser Nullwert wurde von den gemessenen Werten der in den Versuchen exponierten Augendosimeter abgezogen. Bei einer sehr geringen Röntgenexposition (niedrige Zahl der Röntgenaufnahmen bzw. kurze Durchleuchtungszeit) lagen Nullwert und gemessene Untersuchungswerte sehr nah beieinander; durch die Messungenauigkeit der Augendosimeter bei Einzelmessungen konnten so auch unlogisch erscheinende negative Werte entstehen. 


\subsection{Untersuchungen am Phantommodell}

Die Untersuchung der Strahlenbelastung der Augenlinse erfolgte zunächst an einem reproduzierbaren Phantommodell mit standardisierter Strahlenbelastung, um eine Vergleichbarkeit der Messreihen mit und ohne Strahlenschutzvisier zu gewährleisten.

Um einen Untersuchungsaufbau zu schaffen, der den realen Bedingungen bei einer Ureterorenoskopie möglichst nahe kommt, wurde ein sogenanntes AldersonPhantom verwendet und anstelle des Patienten auf dem Röntgentisch positioniert (Abb.6). Das Alderson-Phantom hat die Form eines menschlichen Torsos und führt bei der Röntgenaufnahme bzw. bei der Röntgendurchleuchtung zur einer vergleichbaren Streustrahlung wie der menschliche Körper.

Als Kopf des Operateurs diente ein wassergefüllter Luftballon, dessen Wasserfüllung die Streustrahlung des menschlichen Kopfes widerspiegeln sollte. Dieses Kopfphantom wurde auf einem höhenverstellbaren Tisch mithilfe von Schaumstoffunterlagen in $164 \mathrm{~cm}$ Höhe positioniert (= Kopfhöhe des Operateurs unter den realen Untersuchungsbedingungen). Als Abstand zur Röntgenröhre wurden 0,5 m gewählt, was in etwa dem Abstand des Operateurs von der Röntgenröhre unter realen Untersuchungsbedingungen entspricht.

Das Augendosimeter selbst wurde mithilfe eines elastischen Stirnbandes lateral des rechten Auges positioniert und befestigt, analog der in der Produktbeschreibung vorgegebenen Positionierung des Augendosimeters unter Realbedingungen.

Für die Messreihen mit Strahlenschutzvisier wurde das Strahlenschutzvisier BRV 501 der Firma MAVIG, München, Deutschland verwendet, das so am Kopfphantom befestigt wurde, dass es das Augendosimeter vollständig abdeckte (Abb.7). 
Abbildung 6

Versuchsaufbau mit Alderson-Phantom und Positionierung des Kopfmodells in einer Höhe von $164 \mathrm{~cm}$, ca. 0,5 m von der Röntgenröhre entfernt.

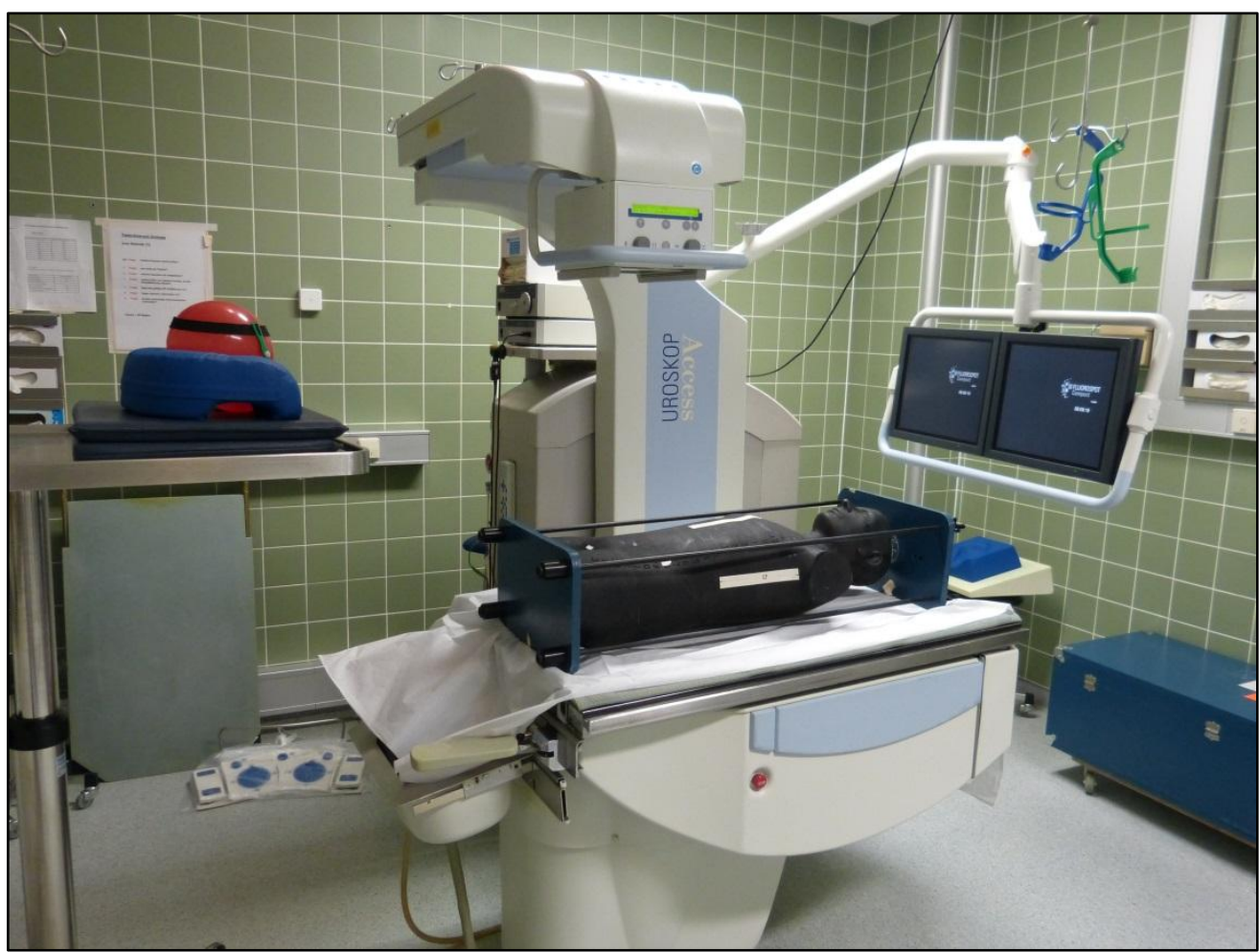

\section{Abbildung 7}

Ballon-Kopfphantom mit fixiertem Thermolumineszenz-Augenlinsendosimeter Eye$D^{\circledR}$, ohne und mit Strahlenschutzvisier (BRV 501, Fa. MAVIG, München, Deutschland)
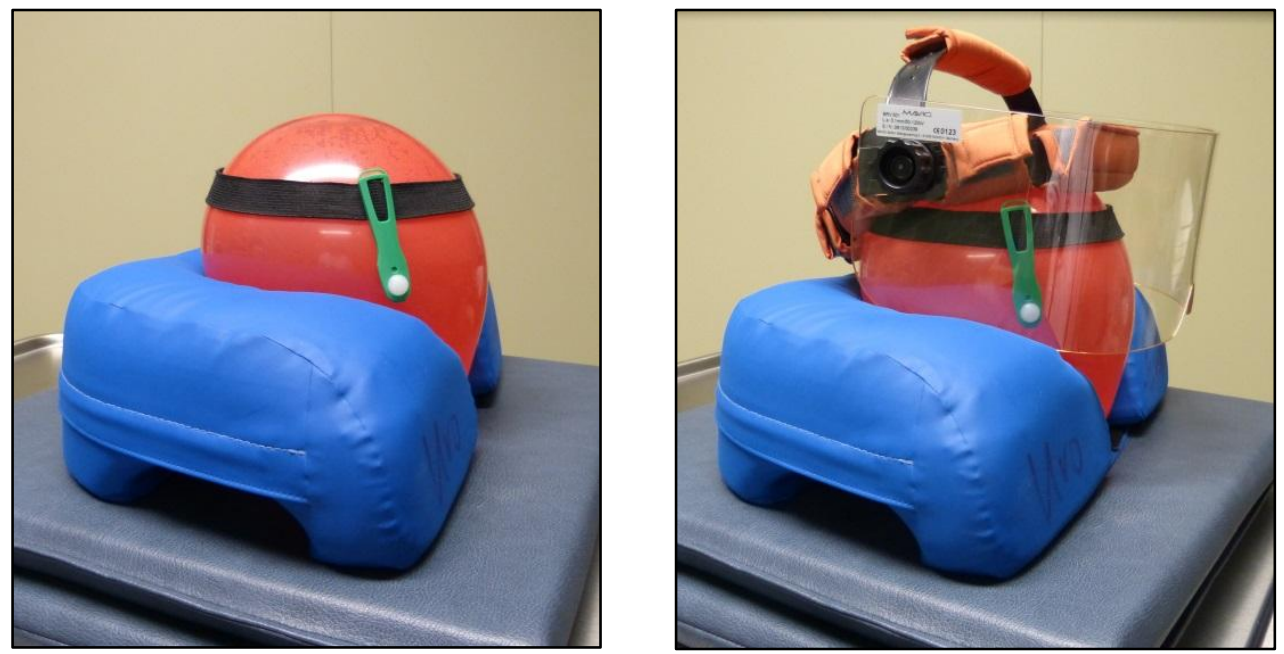
Es wurden folgende 4 Messreihen durchgeführt:

- 1. Messreihe

Messung der Strahlenbelastung am Augendosimeter ohne Strahlenschutzvisier bei zunehmender Zahl an Röntgenaufnahmen mit 1, 2, 3, 4, 5, 6, 7, 8, 9 und 10 Röntgenaufnahmen;

- 2. Messreihe

Messung der Strahlenbelastung am Augendosimeter mit Strahlenschutzvisier bei zunehmender Zahl an Röntgenaufnahmen mit 1, 2, 3, 4, 5, 6, 7, 8, 9 und 10 Röntgenaufnahmen;

- 3. Messreihe

Messung der Strahlenbelastung am Augendosimeter ohne Strahlenschutzvisier bei zunehmender Durchleuchtungszeit von 15, 30, 45, 60, 75, 90, 105, 120, 135, 150, 165 und 180 Sekunden;

\section{- 4. Messreihe}

Messung der Strahlenbelastung am Augendosimeter mit Strahlenschutzvisier bei zunehmender Durchleuchtungszeit von 15, 30, 45, 60, 75, 90, 105, 120, 135, 150, 165 und 180 Sekunden.

Dabei wurden je Messpunkt pro Messreihe 5 Messungen durchgeführt.

Die dabei abgegebene Strahlendosis wurde als Dosisflächenprodukt durch die Messkammer im Strahlengang dokumentiert. Aus den jeweils 5 dokumentierten Messwerten für das Dosisflächenprodukt wurden der arithmetische Mittelwert sowie die Standardabweichung berechnet.

Die Augendosimeter wurden nach jeder Einzelmessung zur Auswertung an die Auswertungsstelle für Strahlendosimeter des Helmholtz Zentrums München geschickt. Die dort gemessenen Strahlenbelastungen pro Augendosimeter wurden dokumentiert. Von den 5 dokumentierten Messergebnissen pro Messreihenpunkt wurden dann zunächst wie beschrieben die zugehörigen Nullwerte subtrahiert, um den Einfluss der natürlichen Strahlenbelastung weitgehend zu minimieren. 
Anschließend wurde aus den errechneten Messergebnissen wiederum der arithmetische Mittelwert mit Standardabweichung berechnet.

Insgesamt wurden damit 220 Einzeluntersuchungen im standardisierten Untersuchungsmodell durchgeführt. 


\subsection{Untersuchungen unter Realbedingungen}

Zusätzlich zu den Untersuchungen im standardisierten Untersuchungsmodell wurden im Zeitraum von 11/2013 bis 10/2014 Untersuchungen zur Strahlenbelastung des Auges unter den Realbedingungen einer ureterorenoskopischen Steinentfernung durchgeführt. Die operativen Eingriffe erfolgten ausschließlich durch die Promovendin, Messungen bei weiteren Operateuren erfolgten nicht.

Insgesamt wurden von der Promovendin 45 ureterorenoskopische Steinentfernungen durchgeführt, davon 22 Eingriffe ohne Strahlenschutzvisier und 23 Eingriffe unter Verwendung des Strahlenschutzvisiers BRV 501 der Fa. MAVIG. Die Zuordnung der Eingriffe zur Durchführung mit oder ohne Strahlenschutzvisier erfolgte vor Beginn der Untersuchungsreihe mittels eines internetbasierten Zufallsgenerators. Das Augendosimeter wurde dabei entsprechend den Herstellervorgaben wie oben beschrieben mittels Trageband lateral des rechten Auges des Operateurs getragen.

Bei jedem Eingriff wurde zur Messung der Strahlendosis das Dosisflächenprodukt dokumentiert. Das Augendosimeter wurde nach jedem Eingriff gewechselt, die Auswertung der Augendosimeter erfolgte wiederum in der Auswertungsstelle des Helmholtz Zentrums in München.

Auch bei diesen Messwerten erfolgte zur Minimierung des Einflusses der natürlichen Strahlenbelastung die Subtraktion eines ermittelten Nullwertes. 


\subsection{Datenerhebung, Datenauswertung und statistische Methoden}

Die für die unterschiedlichen Untersuchungen im Röntgensystem Uroskop Access $^{\circledR}$ mittels einer im Strahlengang eingebrachten Messkammer als Dosisflächenprodukt gemessene Strahlendosis und die mittels des Thermolumineszenz-Augenlinsendosimeters Eye- $D^{\circledR}$ gemessene korrespondierende Augenlinsendosis wurden im Tabellenkalkulationsprogramm Excel 2003 for Windows bzw. Excel 2007 for Windows (Fa. Microsoft, USA) dokumentiert. In diesem Tabellen-Kalkulationsprogramm erfolgte dann die Berechnung der Strahlendosis abzüglich des oben dargestellten „Nullwertes“, die Berechnung der Mittelwerte und der Standardabweichung, Trendberechnungen für den Vergleich der Untersuchung ohne und mit Strahlenschutzvisier unter den realen Bedingungen einer ureterorenoskopischen Steinentfernung. Ebenso wurden im Tabellenkalkulationsprogramm alle graphischen Darstellungen mit Ausnahme der Box-plot-Darstellungen erstellt. Für den statistischen Vergleich der Messergebnisse unter den Bedingungen einer realen ureterorenoskopischen Steinentfernung wurden die Messergebnisse in das Statistikprogramm GraphPad Prism 5 (Fa. GraphPad Inc., La Jolla, USA) überführt. Als statistisches Verfahren kam der t-Test für unverbundene Stichproben zum Einsatz, wobei p-Werte $<0,05$ als statistisch signifikant angesehen wurden. Ebenso wurden mit GraphPad Prism 5 die Box-plot-Graphiken erstellt (Darstellung: minimaler Wert, maximaler Wert, Mittelwert, Box-plot $=$ Werte zwischen der $25-75 \%$ Perzentile). 


\section{Ergebnisse}

\subsection{Messergebnisse am Phantom}

3.1.1 Messungen mit zunehmender Anzahl an Röntgenbildern ohne Strahlenschutzvisier

Mit zunehmender Anzahl an Röntgenbildern stieg die im Strahlengang als Dosisflächenprodukt gemessene Strahlendosis weitgehend linear von 13,0 \pm 0,1 $\mu \mathrm{Gy}^{*} \mathrm{~m}^{2}$ (Mittelwert \pm Standardabweichung) bei einer Röntgenaufnahme auf 129,4 \pm 0,6 $\mu \mathrm{Gy}^{*} \mathrm{~m}^{2}$ (Mittelwert \pm Standardabweichung) bei 10 Röntgenaufnahmen an. Die am Augendosimeter gemessene Strahlendosis folgte dieser Zunahme des gemessenen Dosisflächenprodukts mit einem Anstieg von 0,002 \pm 0,005 mSv (Mittelwert \pm Standardabweichung) bei einer Röntgenaufnahme auf 0,008 \pm 0,003 mSv (Mittelwert \pm Standardabweichung) bei 10 Röntgenaufnahmen (Tab.4 und Abb.8).

\subsubsection{Messungen mit zunehmender Anzahl an Röntgenbildern} mit Strahlenschutzvisier

Auch hier stieg erwartungsgemäß mit zunehmender Anzahl an Röntgenbildern die im Strahlengang als Dosisflächenprodukt gemessene Strahlendosis weitgehend linear von 13,0 $\pm 0,1 \mu \mathrm{Gy}^{*} \mathrm{~m}^{2}$ (Mittelwert \pm Standardabweichung) bei einer Röntgenaufnahme auf 130,0 $\pm 1,1 \mu \mathrm{Gy}^{*} \mathrm{~m}^{2}$ (Mittelwert \pm Standardabweichung) bei 10 Röntgenaufnahmen an. Die am Augendosimeter bemessene Strahlendosis folgte dieser Zunahme allerdings nicht, sondern schwankte zwischen einem Wert von -0,002 $\pm 0,005 \mathrm{mSv}$ (Mittelwert \pm Standardabweichung) bei einer Röntgenaufnahme und $-0,001 \pm 0,003 \mathrm{mSv}$ (Mittelwert \pm Standardabweichung) bei 10 Röntgenaufnahmen (Tab.5 und Abb.9). 


\section{$\underline{\text { Tabelle } 4}$}

Augenlinsendosis bei Aufnahme von Röntgenbildern ohne Strahlenschutzvisier

\begin{tabular}{|c|c|c|}
\hline $\begin{array}{c}\text { Röntgen- } \\
\text { bilder }\end{array}$ & $\begin{array}{c}\text { Dosisflächenprodukt }\left[\mu \mathrm{Gy}^{*} \mathrm{~m}^{2}\right] \\
\text { Mittelwert } \pm \text { Standardabweichung }\end{array}$ & $\begin{array}{c}\text { Augenlinsendosis } \mathrm{Hp}(3)[\mathrm{mSv}] \\
\text { Mittelwert } \pm \text { Standardabweichung }\end{array}$ \\
\hline 1 & $13,0 \pm 0,1$ & $0,002 \pm 0,005$ \\
\hline 2 & $25,8 \pm 0,1$ & $0,003 \pm 0,004$ \\
\hline 3 & $38,7 \pm 0,2$ & $0,004 \pm 0,003$ \\
\hline 4 & $51,7 \pm 0,4$ & $0,004 \pm 0,007$ \\
\hline 5 & $64,8 \pm 0,3$ & $0,006 \pm 0,004$ \\
\hline 6 & $77,6 \pm 0,5$ & $0,008 \pm 0,008$ \\
\hline 7 & $90,5 \pm 0,4$ & $0,011 \pm 0,006$ \\
\hline 8 & $103,8 \pm 0,4$ & $0,009 \pm 0,005$ \\
\hline 9 & $116,5 \pm 0,7$ & $0,010 \pm 0,004$ \\
\hline 10 & $129,4 \pm 0,6$ & $0,008 \pm 0,003$ \\
\hline
\end{tabular}

Abbildung 8

Augenlinsendosis bei Aufnahme von Röntgenbildern ohne Strahlenschutzvisier (Mittelwerte)

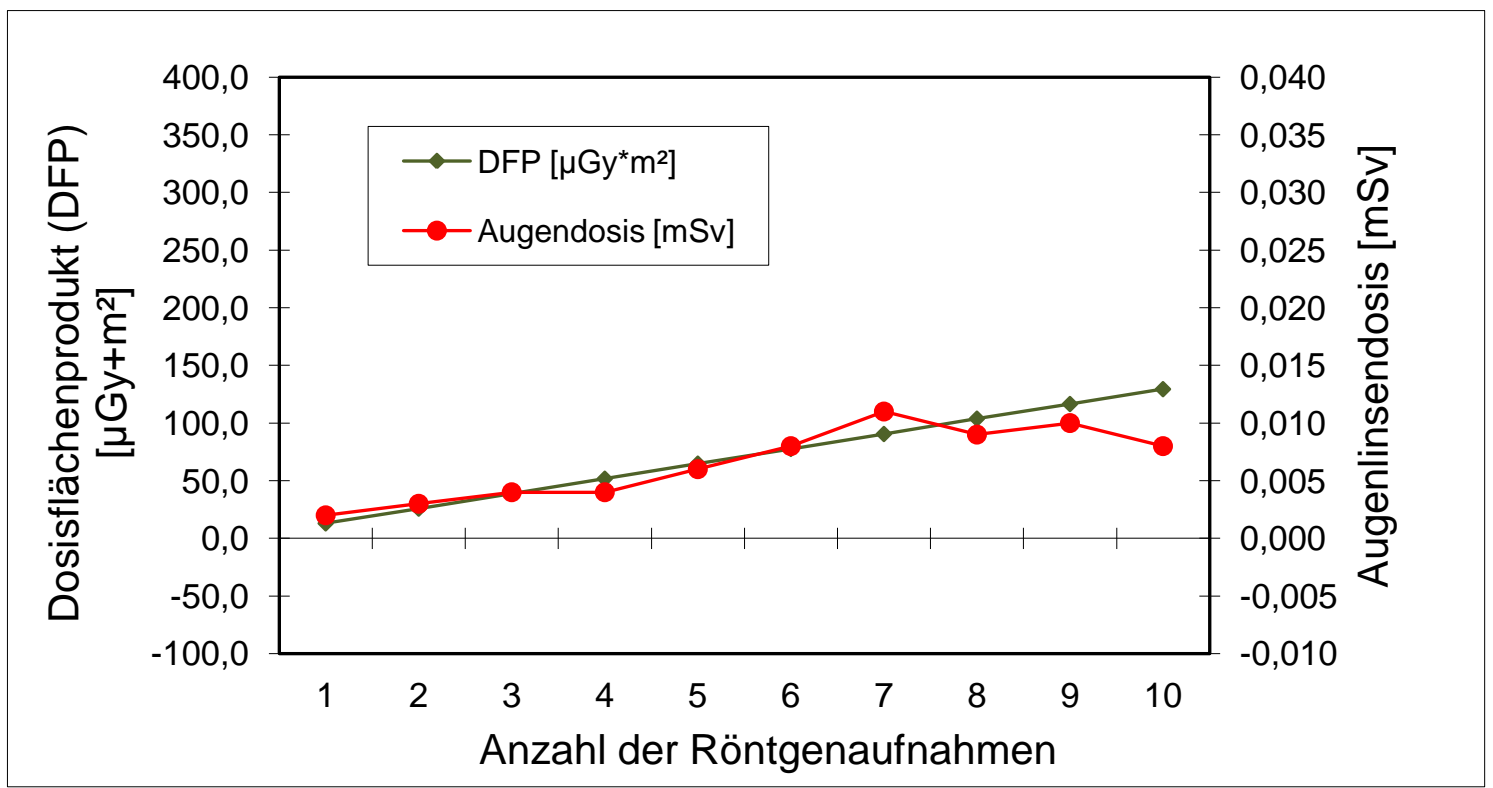




\section{$\underline{\text { Tabelle } 5}$}

Augenlinsendosis bei Aufnahme von Röntgenbildern mit Strahlenschutzvisier

\begin{tabular}{|c|c|c|}
\hline $\begin{array}{c}\text { Röntgen- } \\
\text { bilder }\end{array}$ & $\begin{array}{c}\text { Dosisflächenprodukt }\left[\mathrm{GG}^{*} \mathrm{~m}^{2}\right] \\
\text { Mittelwert } \pm \text { Standardabweichung }\end{array}$ & $\begin{array}{c}\text { Augenlinsendosis } \mathrm{Hp}(3)[\mathrm{mSv}] \\
\text { Mittelwert } \pm \text { Standardabweichung }\end{array}$ \\
\hline 1 & $13,0 \pm 0,1$ & $-0,002 \pm 0,005$ \\
\hline 2 & $26,3 \pm 0,6$ & $-0,002 \pm 0,004$ \\
\hline 3 & $39,0 \pm 0,1$ & $-0,003 \pm 0,001$ \\
\hline 4 & $51,1 \pm 0,3$ & $-0,001 \pm 0,004$ \\
\hline 5 & $64,8 \pm 0,4$ & $0,001 \pm 0,007$ \\
\hline 6 & $78,0 \pm 0,7$ & $-0,001 \pm 0,006$ \\
\hline 7 & $90,9 \pm 0,5$ & $0,004 \pm 0,005$ \\
\hline 8 & $103,7 \pm 0,4$ & $0,001 \pm 0,005$ \\
\hline 9 & $117,0 \pm 0,5$ & $0,003 \pm 0,004$ \\
\hline 10 & $130,0 \pm 1,1$ & $-0,001 \pm 0,003$ \\
\hline
\end{tabular}

Abbildung 9

Augenlinsendosis bei Aufnahme von Röntgenbildern mit Strahlenschutzvisier (Mittelwerte)

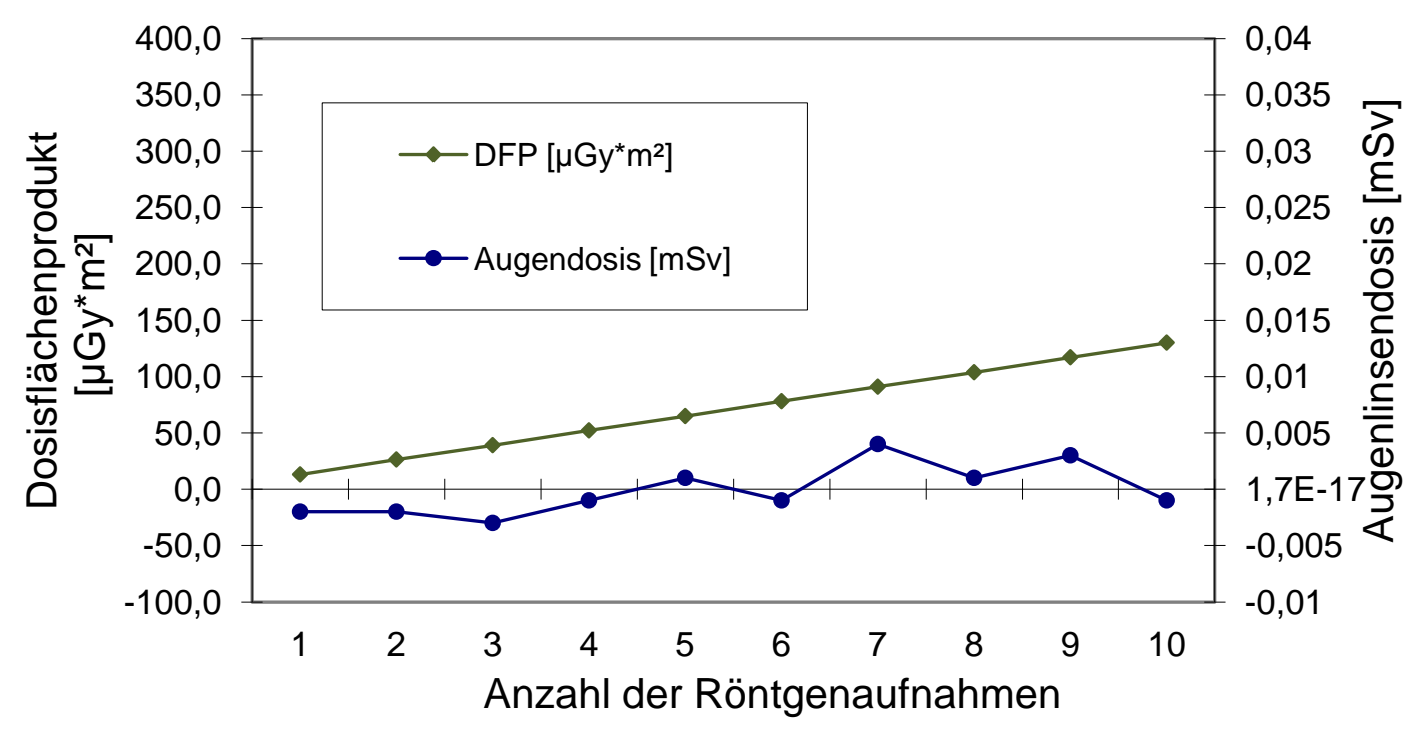




\subsubsection{Messungen mit zunehmender Durchleuchtungszeit}

ohne Strahlenschutzvisier

Mit zunehmender Durchleuchtungszeit stieg die im Strahlengang als Dosisflächenprodukt gemessene Strahlendosis weitgehend linear von 29,6 \pm 0,7 $\mu \mathrm{Gy}^{*} \mathrm{~m}^{2}$ (Mittelwert \pm Standardabweichung) bei einer Durchleuchtungszeit von 15 Sekunden auf 355,1 $\pm 7,4 \mu \mathrm{Gy}^{*} \mathrm{~m}^{2}$ (Mittelwert \pm Standardabweichung) bei einer Durchleuchtungszeit von 180 Sekunden an. Die am Augendosimeter bemessene Strahlendosis folgte dieser Zunahme des gemessenen Dosisflächenprodukts mit einem Anstieg von 0,002 \pm 0,002 mSv (Mittelwert \pm Standardabweichung) bei einer Durchleuchtungszeit von 15 Sekunden auf 0,024 \pm 0,011 mSv (Mittelwert \pm Standardabweichung) bei einer Durchleuchtungszeit 180 Sekunden (Tab.6 und Abb.10).

\subsubsection{Messungen mit zunehmender Durchleuchtungszeit mit Strahlenschutzvisier}

Auch hier stieg mit zunehmender Durchleuchtungszeit die im Strahlengang als Dosisflächenprodukt gemessene Strahlendosis vergleichbar mit den Messungen ohne Strahlenschutzvisier weitgehend linear von 29,7 $\pm 0,5 \mu \mathrm{Gy}^{*} \mathrm{~m}^{2}$ (Mittelwert \pm Standardabweichung) bei einer Durchleuchtungszeit von 15 Sekunden auf 349,7 \pm 6,7 $\mu \mathrm{Gy}^{*} \mathrm{~m}^{2}$ (Mittelwert \pm Standardabweichung) bei einer Durchleuchtungszeit von 180 Sekunden an. Auch hier folgte die am Augendosimeter gemessene Strahlendosis dieser Zunahme nicht. Die gemessene Augenlinsendosis lag zwischen 0,001 \pm 0,002 mSv (Mittelwert \pm Standardabweichung) bei einer Durchleuchtungszeit von 15 Sekunden und 0,000 \pm 0,006 mSv (Mittelwert \pm Standardabweichung) bei einer Durchleuchtungszeit von 180 Sekunden (Tab.7 und Abb.11). 
$\underline{\text { Tabelle } 6}$

Augenlinsendosis bei Durchleuchtung ohne Strahlenschutzvisier

\begin{tabular}{|c|c|c|}
\hline $\begin{array}{c}\text { Durchleuch- } \\
\text { tungszeit }[\mathrm{s}]\end{array}$ & $\begin{array}{c}\text { Dosisflächenprodukt }\left[\mu \mathrm{Gy}^{*} \mathrm{~m}^{2}\right] \\
\text { Mittelwert } \pm \text { Standardabweichung }\end{array}$ & $\begin{array}{c}\text { Augenlinsendosis } \mathrm{Hp}(3)[\mathrm{mSv}] \\
\text { Mittelwert } \pm \text { Standardabweichung }\end{array}$ \\
\hline 15 & $29,6 \pm 0,7$ & $0,002 \pm 0,002$ \\
\hline 30 & $58,9 \pm 1,4$ & $0,007 \pm 0,005$ \\
\hline 45 & $89,0 \pm 0,8$ & $0,008 \pm 0,005$ \\
\hline 60 & $118,4 \pm 0,7$ & $0,006 \pm 0,004$ \\
\hline 75 & $145,7 \pm 3,2$ & $0,010 \pm 0,008$ \\
\hline 90 & $176,7 \pm 2,7$ & $0,010 \pm 0,001$ \\
\hline 105 & $204,8 \pm 3,8$ & $0,010 \pm 0,004$ \\
\hline 120 & $236,5 \pm 2,6$ & $0,017 \pm 0,006$ \\
\hline 135 & $263,2 \pm 4,9$ & $0,016 \pm 0,006$ \\
\hline 150 & $295,3 \pm 4,7$ & $0,018 \pm 0,011$ \\
\hline 165 & $324,8 \pm 6,2$ & $0,024 \pm 0,013$ \\
\hline 180 & $355,1 \pm 7,4$ & $0,024 \pm 0,011$ \\
\hline
\end{tabular}

Abbildung 10

Augenlinsendosis bei Durchleuchtung ohne Strahlenschutzvisier (Mittelwerte)

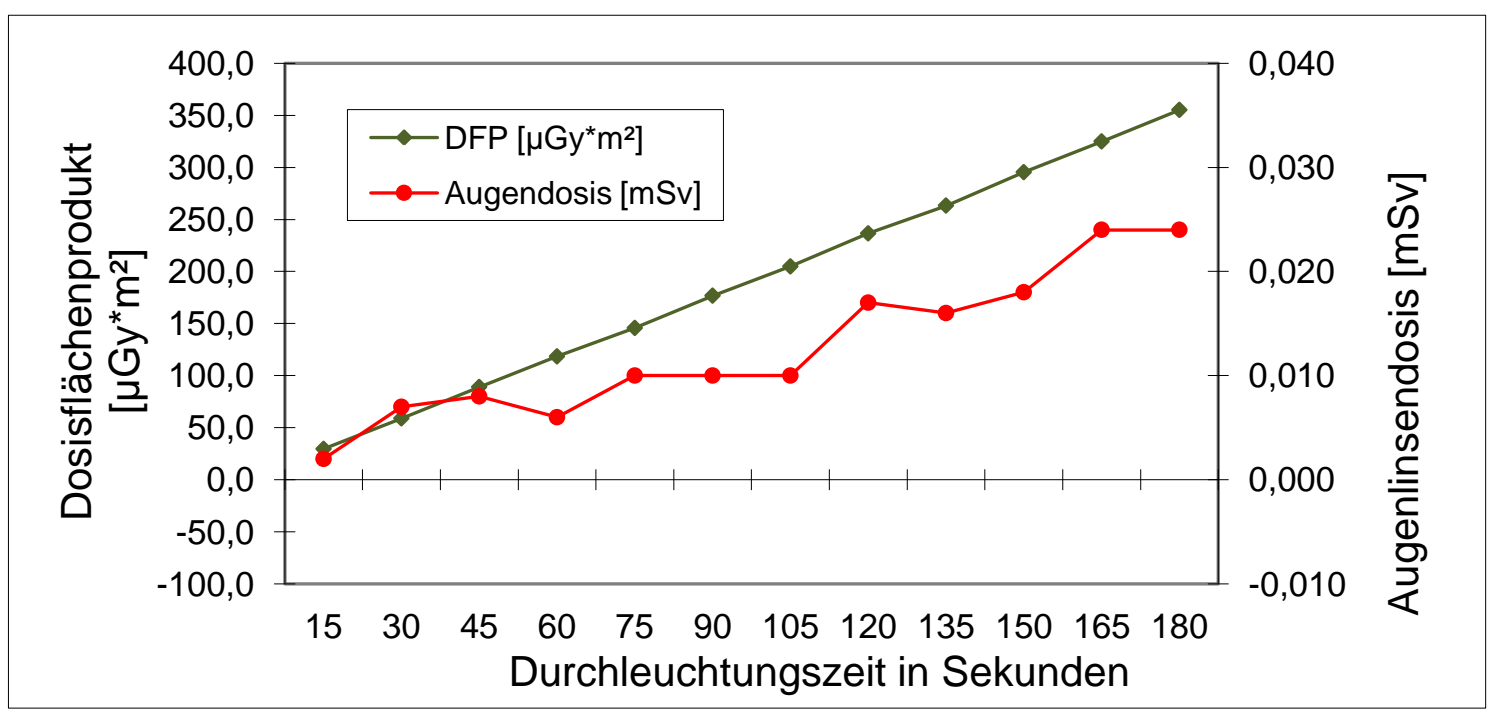


$\underline{\text { Tabelle } 7}$

Augenlinsendosis bei Durchleuchtung mit Strahlenschutzvisier

\begin{tabular}{|c|c|c|}
\hline $\begin{array}{c}\text { Durchleuch- } \\
\text { tungszeit }[\mathrm{s}]\end{array}$ & $\begin{array}{c}\text { Dosisflächenprodukt }\left[\mu \mathrm{Gy}^{*} \mathrm{~m}^{2}\right] \\
\text { Mittelwert } \pm \text { Standardabweichung }\end{array}$ & $\begin{array}{c}\text { Augenlinsendosis } \mathrm{Hp}(3)[\mathrm{mSv}] \\
\text { Mittelwert } \pm \text { Standardabweichung }\end{array}$ \\
\hline 15 & $29,7 \pm 0,5$ & $0,001 \pm 0,002$ \\
\hline 30 & $59,7 \pm 0,6$ & $-0,002 \pm 0,002$ \\
\hline 45 & $89,7 \pm 1,7$ & $0,000 \pm 0,003$ \\
\hline 60 & $118,3 \pm 1,7$ & $-0,002 \pm 0,004$ \\
\hline 75 & $146,8 \pm 2,0$ & $0,001 \pm 0,003$ \\
\hline 90 & $176,6 \pm 3,8$ & $0,002 \pm 0,004$ \\
\hline 105 & $206,9 \pm 3,7$ & $-0,002 \pm 0,002$ \\
\hline 120 & $236,7 \pm 5,3$ & $0,003 \pm 0,005$ \\
\hline 135 & $261,1 \pm 5,6$ & $0,003 \pm 0,005$ \\
\hline 150 & $293,5 \pm 6,3$ & $0,002 \pm 0,004$ \\
\hline 165 & $321,9 \pm 6,7$ & $0,000 \pm 0,002$ \\
\hline 180 & $349,7 \pm 6,7$ & $0,000 \pm 0,006$ \\
\hline
\end{tabular}

Abbildung 11

Augenlinsendosis bei Durchleuchtung mit Strahlenschutzvisier (Mittelwerte)

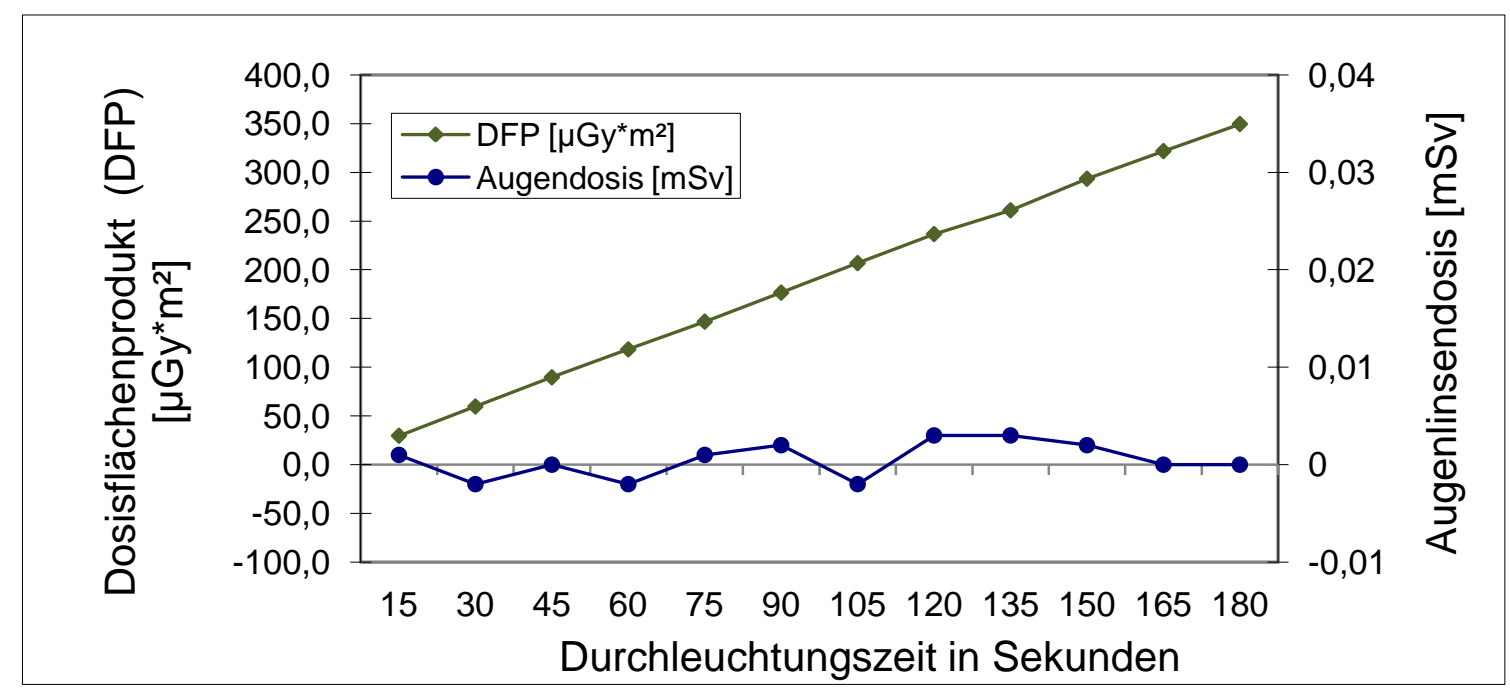




\subsubsection{Vergleich der Messungen ohne und mit Strahlenschutzvisier}

Die Untersuchung konnte somit zeigen, dass die Strahlenbelastung am Augendosimeter unter standardisierten Untersuchungsbedingungen durch die Verwendung eines Strahlenschutzvisiers gesenkt werden kann. Der Effekt nimmt dabei mit zunehmender Strahlenexposition zu.

Die am Augendosimeter gemessene Belastung lag bei einer Röntgenaufnahme ohne Strahlenschutzvisier 0,002 \pm 0,005 mSv (Mittelwert \pm Standardabweich-ung), mit Strahlenschutzvisier bei $-0,002 \pm 0,005 \mathrm{mSv}$ (Mittelwert \pm Standardabweichung. Bei 10 Röntgenaufnahmen lag die Strahlenbelastung ohne Strahlenschutzvisier bei 0,008 \pm 0,003 mSv (Mittelwert \pm Standardabweichung), mit Strahlenschutzvisier bei $-0,001 \pm 0,003 \mathrm{mSv}$ (Mittelwert \pm Standardabweichung) (Abb.12). Die im Strahlengang gemessene Strahlendosis war dabei vergleichbar mit jeweils 13,0 $\pm 0,1 \mu \mathrm{Gy}^{*} \mathrm{~m}^{2}$ (Mittelwert \pm Standardabweichung) bei einer Röntgenaufnahme und 129,4 \pm 0,6 $\mu \mathrm{Gy}^{*} \mathrm{~m}^{2}$ bzw. 130,0 $\pm 1,1 \mu \mathrm{Gy}^{*} \mathrm{~m}^{2}$ (Mittelwert \pm Standardabweichung) bei 10 Röntgenaufnahmen.

Bei der Untersuchungsreihe mit steigender Durchleuchtungszeit lag die Strahlenbelastung bei einer Durchleuchtungszeit von 15 Sekunden ohne Strahlenschutzvisier bei 0,002 \pm 0,002 mSv (Mittelwert \pm Standardabweichung), mit Strahlenschutzvisier bei 0,001 \pm 0,002 mSv (Mittelwert \pm Standardabweichung). Bei einer Durchleuchtungszeit von 180 Sekunden stieg die gemessene Augenlinsendosis ohne Strahlenschutzvisier auf 0,024 \pm 0,011 mSv (Mittelwert \pm Standardabweichung), mit Strahlenschutzvisier betrug sie 0,000 $\pm 0,006 \mathrm{mSv}$ (Mittelwert \pm Standardabweichung) (Abb.13). Auch hier war die im Strahlengang gemessene Strahlendosis mit einem Dosisflächenprodukt von jeweils 29,8 \pm 0,7 $\mu \mathrm{Gy}^{*} \mathrm{~m}^{2}$ bzw. 29,7 \pm 0,5 (Mittelwert \pm Standardabweichung) bei einer Durchleuchtungszeit von 15 Sekunden und 355,1 \pm 7,4 $\mu \mathrm{Gy}^{*} \mathrm{~m}^{2}$ bzw. $349,7 \pm 6,7$ $\mu \mathrm{Gy}^{*} \mathrm{~m}^{2}$ (Mittelwert \pm Standardabweichung) bei einer Durchleuchtungszeit von 180 Sekunden vergleichbar. 
Abbildung 12

Reduktion der Strahlenbelastung unter Verwendung eines Strahlenschutzvisiers im Vergleich zur Strahlenbelastung ohne Strahlenschutzvisier bei Röntgenaufnahmen (Mittelwerte).

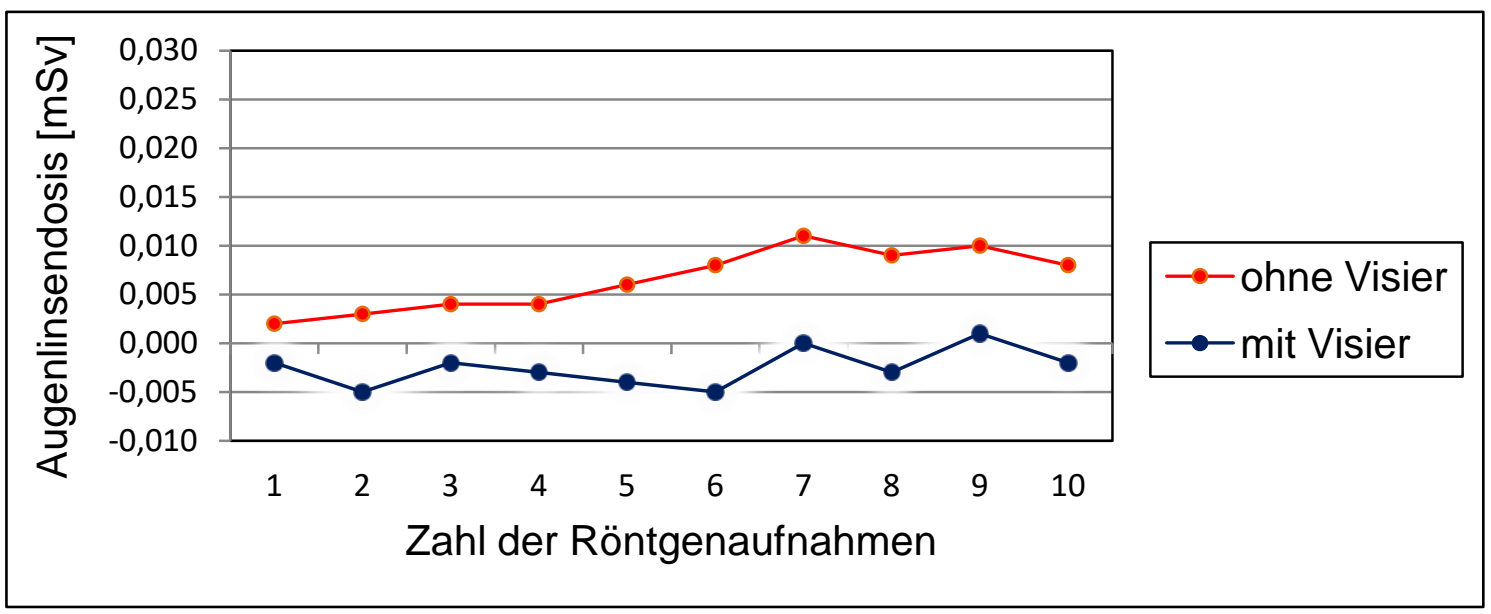

\section{Abbildung 13}

Reduktion der Strahlenbelastung unter Verwendung eines Strahlenschutzvisiers im Vergleich zur Strahlenbelastung ohne Strahlenschutzvisier bei der Durchleuchtung (Mittelwerte).

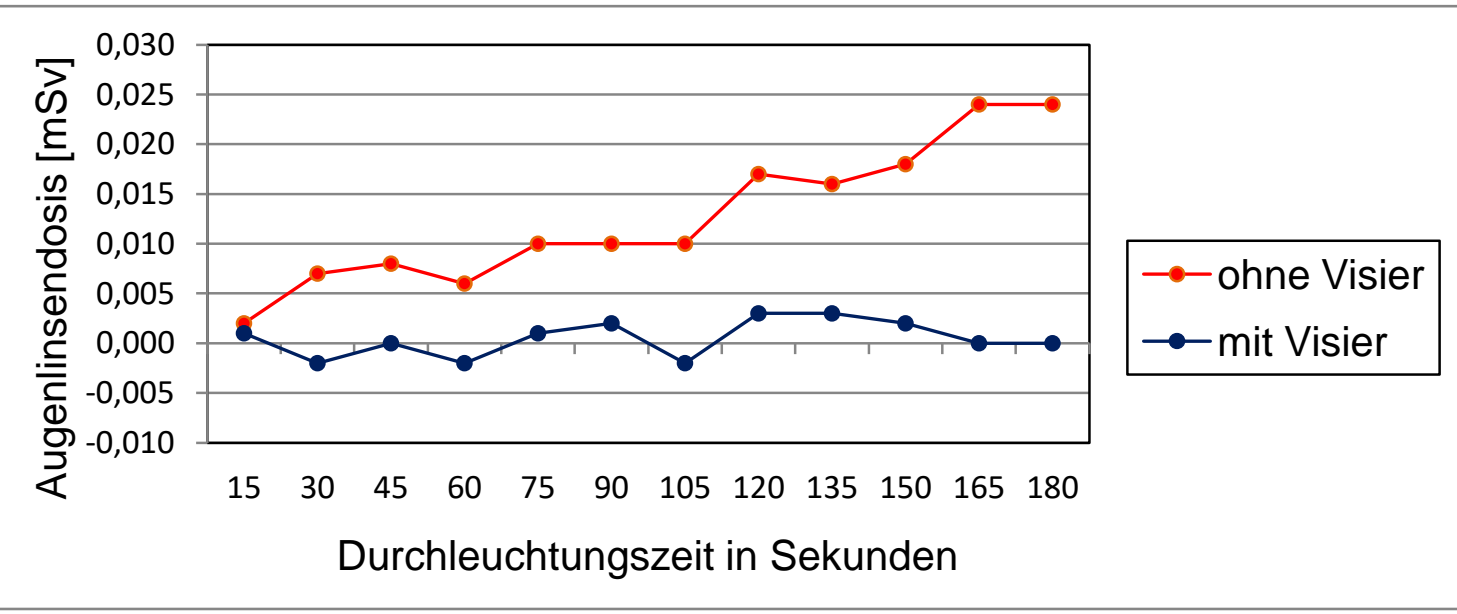


Die Streubreite der gemessenen Augenlinsendosis war dabei aber insbesondere bei geringer Strahlenbelastung so groß, dass beim Vergleich von Röntgenaufnahmen nur in 2 von 10 Untersuchungsreihen ein statistisches Signifikanzniveau von $<0,05$ (t-Test) erreicht wurde (Tab.8). Deutlicher fielen die Unterschiede in der Untersuchungsreihe mit steigender Durchleuchtungszeit aus. Hier fand sich bei der Verwendung eines Strahlenschutzvisiers ab einer Durchleuchtungszeit von 30 Sekunden mit einer Ausnahme (75 Sekunden) eine durchgehende statistisch signifikante Reduzierung der Augenlinsendosis (Tab.9.). 
$\underline{\text { Tabelle } 8}$

Augenlinsendosis bei zunehmender Zahl der Röntgenaufnahmen im Vergleich ohne und mit Strahlenschutzvisier (Mittelwert \pm Standardabweichung)

\begin{tabular}{|c|c|c|c|}
\hline $\begin{array}{c}\text { Röntgen- } \\
\text { bilder }\end{array}$ & $\begin{array}{c}\text { ohne Strahlenschutzvisier } \\
\text { Augenlinsendosis }[\mathrm{mSv}]\end{array}$ & $\begin{array}{c}\text { mit Strahlenschutzvisier } \\
\text { Augenlinsendosis }[\mathrm{mSv}]\end{array}$ & $\begin{array}{c}\text { Signifikanzniveau } \\
\mathrm{t}-\text { Test }\end{array}$ \\
\hline 1 & $0,002 \pm 0,005$ & $-0,002 \pm 0,005$ & $\mathrm{p}=0,3698$ \\
\hline 2 & $0,003 \pm 0,004$ & $-0,002 \pm 0,004$ & $\mathrm{p}=0,9496$ \\
\hline 3 & $0,004 \pm 0,003$ & $-0,003 \pm 0,001$ & $\mathrm{p}=0,0029$ \\
\hline 4 & $0,004 \pm 0,007$ & $-0,001 \pm 0,004$ & $\mathrm{p}=0,3017$ \\
\hline 5 & $0,006 \pm 0,004$ & $0,001 \pm 0,007$ & $\mathrm{p}=0,2493$ \\
\hline 6 & $0,008 \pm 0,008$ & $-0,001 \pm 0,006$ & $\mathrm{p}=0,1118$ \\
\hline 7 & $0,011 \pm 0,006$ & $0,004 \pm 0,005$ & $\mathrm{p}=0,1083$ \\
\hline 8 & $0,009 \pm 0,005$ & $0,001 \pm 0,005$ & $\mathrm{p}=0,0535$ \\
\hline 9 & $0,010 \pm 0,004$ & $0,003 \pm 0,004$ & $\mathrm{p}=0,0642$ \\
\hline 10 & $0,008 \pm 0,003$ & $-0,001 \pm 0,003$ & $\mathrm{p}=0,0015$ \\
\hline
\end{tabular}

$\underline{\text { Tabelle } 9}$

Augenlinsendosis bei zunehmender Durchleuchtungszeit im Vergleich ohne und mit Strahlenschutzvisier (Mittelwert \pm Standardabweichung)

\begin{tabular}{|c|c|c|c|}
\hline $\begin{array}{c}\text { Durchleuch- } \\
\text { tungszeit [s] }\end{array}$ & $\begin{array}{c}\text { ohne Strahlenschutzvisier } \\
\text { Augenlinsendosis [mSv] }\end{array}$ & $\begin{array}{c}\text { mit Strahlenschutzvisier } \\
\text { Augenlinsendosis }[\mathrm{mSv}]\end{array}$ & $\begin{array}{c}\text { Signifikanzniveau } \\
\text { t-Test }\end{array}$ \\
\hline 15 & $0,002 \pm 0,002$ & $0,001 \pm 0,002$ & $\mathrm{p}=0,4419$ \\
\hline 30 & $0,007 \pm 0,005$ & $-0,002 \pm 0,002$ & $\mathrm{p}=0,0107$ \\
\hline 45 & $0,008 \pm 0,005$ & $0,000 \pm 0,003$ & $\mathrm{p}=0,0248$ \\
\hline 60 & $0,006 \pm 0,004$ & $-0,002 \pm 0,004$ & $\mathrm{p}=0,0177$ \\
\hline 75 & $0,010 \pm 0,008$ & $0,001 \pm 0,003$ & $\mathrm{p}=0,1014$ \\
\hline 90 & $0,010 \pm 0,001$ & $0,002 \pm 0,004$ & $\mathrm{p}=0,0066$ \\
\hline 105 & $0,010 \pm 0,004$ & $-0,002 \pm 0,002$ & $\mathrm{p}=0,0009$ \\
\hline 120 & $0,017 \pm 0,006$ & $0,003 \pm 0,005$ & $\mathrm{p}=0,0061$ \\
\hline 135 & $0,016 \pm 0,006$ & $0,003 \pm 0,005$ & $\mathrm{p}=0,0097$ \\
\hline 150 & $0,018 \pm 0,011$ & $0,002 \pm 0,004$ & $\mathrm{p}=0,0202$ \\
\hline 165 & $0,024 \pm 0,013$ & $0,000 \pm 0,002$ & $\mathrm{p}=0,0097$ \\
\hline 180 & $0,024 \pm 0,011$ & $0,000 \pm 0,006$ & $\mathrm{p}=0,0052$ \\
\hline
\end{tabular}




\subsection{Messergebnisse unter Realbedingungen}

Die Zuordnung der Eingriffe bzgl. der Verwendung eines Strahlenschutzvisiers erfolgte mittels eines internetbasierten Zufallsgenerators. Es wurde somit keine Vorauswahl bezüglich der Steingröße und -lokalisation und somit zu erwartender Dauer des Eingriffs getroffen (Tab.10). 22 Eingriffe erfolgten ohne Strahlenschutzvisier, 23 Eingriffe unter Verwendung eines Strahlenschutzvisiers. Bei einer Messung mit Visier konnte die Augenlinsendosis aufgrund eines Materialdefekts des Augendosimeters nicht bestimmt werden, sodass jeweils 22 auswertbare Eingriffe mit und ohne Visier berücksichtigt wurden.

Bei den 22 Eingriffen ohne Strahlenschutzvisier betrug die Durchleuchtungszeit zwischen 7 und 123 Sekunden (Mittelwert 27,7 Sekunden) und die Zahl der durchgeführten Röntgenaufnahmen 0 bis 2 (Mittelwert 0,1). Hierdurch ergab sich eine Strahlenbelastung gemessen als Dosisflächenprodukt im Strahlengang von 4,2 - 294,0 $\mu \mathrm{Gy}^{*} \mathrm{~m}^{2}$ (Mittelwert 44,3 $\mu \mathrm{Gy}^{*} \mathrm{~m}^{2}$ ) (Tab.11).

Bei den 22 Eingriffen unter Verwendung eines Strahlenschutzvisiers betrug die Durchleuchtungszeit zwischen 7 und 273 Sekunden (Mittelwert 52,2 Sekunden), die Zahl der durchgeführten Röntgenaufnahmen 0 - 9 (Mittelwert 0,8). Hierdurch ergab sich eine Strahlenbelastung gemessen als Dosisflächenprodukt im Strahlengang von 3,8 bis 487,8 $\mu \mathrm{Gy}^{*} \mathrm{~m}^{2}$ (Mittelwert $111,4 \mu G y^{*} \mathrm{~m}^{2}$ ) (Tab.12).

Statistisch messbare Unterschiede zwischen den Messungen ohne Strahlenschutzvisier und den Messungen unter Verwendung eines Strahlenschutzvisiers waren aber trotz der unterschiedlichen Mittelwerte nicht belegbar (Tab.13). 
$\underline{\text { Tabelle } 10}$

Strahlendosismessung unter Realbedingungen mit prospektiver Zufallsreihung

\begin{tabular}{|c|c|c|c|c|c|c|}
\hline Messung & $\begin{array}{l}\text { ohne (0) } \\
\text { mit (1) } \\
\text { Visier }\end{array}$ & $\begin{array}{l}\text { Durchleuch- } \\
\text { tungszeit } \\
\text { [sec] }\end{array}$ & $\begin{array}{l}\text { Last- } \\
\text { Image- } \\
\text { Hold }\end{array}$ & $\begin{array}{l}\text { Röntgen- } \\
\text { aufnahmen }\end{array}$ & $\begin{array}{l}\text { Dosisflächen- } \\
\text { produkt } \\
{\left[\mu G y^{*} m^{2}\right]}\end{array}$ & $\begin{array}{l}\text { Augenlinsen- } \\
\text { dosis [mSv] }\end{array}$ \\
\hline 1 & 1 & 7 & 3 & 0 & 11,5 & 0,006 \\
\hline 2 & 1 & 12 & 3 & 0 & 32,3 & 0,016 \\
\hline 5 & 1 & 9 & 5 & 0 & 4,6 & 0,018 \\
\hline 4 & 1 & 15 & 4 & 0 & 22,7 & 0,032 \\
\hline 5 & 0 & 123 & 6 & 0 & 220,0 & 0,016 \\
\hline 6 & 1 & 58 & 9 & 0 & 355,9 & 0,003 \\
\hline 7 & 0 & 22 & 6 & 0 & 66,5 & 0,006 \\
\hline 8 & 1 & 59 & 9 & 1 & 277,9 & 0,013 \\
\hline 9 & 0 & 10 & 3 & 0 & 14,1 & $-0,010$ \\
\hline 10 & 0 & 35 & 6 & 0 & 41,9 & 0,001 \\
\hline 11 & 0 & 32 & 7 & 0 & 31,4 & 0,005 \\
\hline 12 & 1 & 234 & 8 & 4 & 487,8 & 0,001 \\
\hline 13 & 0 & 15 & 3 & 0 & 40,0 & 0,001 \\
\hline 14 & 0 & 12 & 4 & 0 & 6,8 & 0,001 \\
\hline 15 & 1 & 39 & 15 & 0 & 15,0 & $-0,003$ \\
\hline 16 & 0 & 15 & 6 & 0 & 5,6 & 0,003 \\
\hline 17 & 0 & 7 & 3 & 0 & 4,2 & $-0,009$ \\
\hline 18 & 1 & 23 & 4 & 1 & 42,4 & $-0,001$ \\
\hline 19 & 0 & 14 & 6 & 0 & 32,9 & 0,003 \\
\hline 20 & 0 & 17 & 4 & 0 & 9,3 & 0,001 \\
\hline 21 & 0 & 11 & 4 & 0 & 9,3 & $-0,003$ \\
\hline 22 & 0 & 9 & 2 & 0 & 10,9 & 0,018 \\
\hline 23 & 0 & 16 & 6 & 0 & 11,5 & 0,003 \\
\hline 24 & 1 & 7 & 2 & 0 & 6,7 & 0,001 \\
\hline 25 & 1 & 49 & 5 & 0 & 70,3 & 0,007 \\
\hline 26 & 1 & 58 & 4 & 0 & 255,8 & 0,018 \\
\hline 27 & 1 & 25 & 3 & 0 & 33,5 & $\begin{array}{c}\text { Detektor- } \\
\text { defekt }\end{array}$ \\
\hline 28 & 0 & 9 & 2 & 0 & 10,0 & 0,009 \\
\hline 29 & 0 & 66 & 9 & 0 & 58,4 & 0,013 \\
\hline
\end{tabular}




\begin{tabular}{|c|c|c|c|c|c|c|}
\hline 30 & 1 & 34 & 5 & 0 & 74,0 & 0,013 \\
\hline 31 & 1 & 41 & 5 & 0 & 64,2 & 0,017 \\
\hline 32 & 0 & 17 & 4 & 0 & 15,7 & 0,005 \\
\hline 33 & 0 & 121 & 7 & 2 & 294,0 & 0,018 \\
\hline 34 & 0 & 9 & 3 & 0 & 18,4 & 0,003 \\
\hline 35 & 1 & 25 & 7 & 0 & 21,5 & 0,006 \\
\hline 36 & 1 & 69 & 6 & 2 & 116,0 & 0,002 \\
\hline 37 & 1 & 14 & 4 & 0 & 36,2 & 0,012 \\
\hline 38 & 1 & 8 & 5 & 0 & 3,8 & 0,002 \\
\hline 39 & 0 & 30 & 7 & 0 & 42,5 & 0,011 \\
\hline 40 & 0 & 12 & 4 & 0 & 21,1 & $-0,008$ \\
\hline 41 & 1 & 25 & 5 & 0 & 35,1 & 0,008 \\
\hline 42 & 1 & 56 & 6 & 0 & 68,9 & 0,006 \\
\hline 43 & 1 & 34 & 7 & 0 & 38,2 & $-0,003$ \\
\hline 44 & 1 & 273 & 4 & 9 & 409,6 & 0,003 \\
\hline 45 & 0 & 8 & 3 & 0 & 9,0 & 0,013 \\
\hline
\end{tabular}


$\underline{\text { Tabelle } 11}$

Untersuchungsergebnisse unter Realbedingungen ohne Verwendung eines Strahlenschutzvisiers, aufsteigend sortiert nach Dosisflächenprodukt

\begin{tabular}{|c|c|c|c|c|c|}
\hline Messung & $\begin{array}{c}\text { Durchleuch- } \\
\text { tungszeit } \\
\text { [sec] }\end{array}$ & $\begin{array}{c}\text { Last- } \\
\text { Image- } \\
\text { Hold }\end{array}$ & $\begin{array}{c}\text { Röntgen- } \\
\text { bilder }\end{array}$ & $\begin{array}{c}\text { Dosisflächen- } \\
\text { produkt } \\
{\left[\mathrm{GG}^{*} \mathrm{~m}^{2}\right]}\end{array}$ & $\begin{array}{c}\text { Augenlinsen- } \\
\text { dosis [mSv] }\end{array}$ \\
\hline 17 & 7 & 3 & 0 & 4,2 & $-0,009$ \\
\hline 16 & 15 & 6 & 0 & 5,6 & 0,003 \\
\hline 14 & 12 & 4 & 0 & 6,8 & 0,001 \\
\hline 45 & 8 & 3 & 0 & 9,0 & 0,013 \\
\hline 20 & 17 & 4 & 0 & 9,3 & 0,001 \\
\hline 21 & 11 & 4 & 0 & 9,3 & $-0,003$ \\
\hline 28 & 9 & 2 & 0 & 10,0 & 0,009 \\
\hline 22 & 9 & 2 & 0 & 10,9 & 0,018 \\
\hline 23 & 16 & 6 & 0 & 11,5 & 0,003 \\
\hline 9 & 10 & 3 & 0 & 14,1 & $-0,010$ \\
\hline 32 & 17 & 4 & 0 & 15,7 & 0,005 \\
\hline 34 & 9 & 3 & 0 & 18,4 & 0,003 \\
\hline 40 & 12 & 4 & 0 & 21,1 & $-0,008$ \\
\hline 11 & 32 & 7 & 0 & 31,4 & 0,005 \\
\hline 19 & 14 & 6 & 0 & 32,9 & 0,003 \\
\hline 13 & 15 & 3 & 0 & 40,0 & 0,001 \\
\hline 10 & 35 & 6 & 0 & 41,9 & 0,001 \\
\hline 39 & 30 & 7 & 0 & 42,5 & 0,011 \\
\hline 29 & 66 & 9 & 0 & 58,4 & 0,013 \\
\hline 7 & 22 & 6 & 0 & 66,5 & 0,006 \\
\hline 5 & 123 & 6 & 0 & 220,0 & 0,016 \\
\hline 33 & 121 & 7 & 2 & 294,0 & 0,018 \\
\hline & & & & & \\
\hline Mittelwert & 27,7 & 4,7 & 0,2 & 44,3 & 0,005 \\
\hline & & & & & \\
\hline
\end{tabular}


$\underline{\text { Tabelle } 12}$

Untersuchungsergebnisse unter Realbedingungen mit Verwendung eines Strahlenschutzvisiers, aufsteigend sortiert nach Dosisflächenprodukt

\begin{tabular}{|c|c|c|c|c|c|}
\hline Messung & $\begin{array}{c}\text { Durchleuch- } \\
\text { tungszeit } \\
\text { [sec] }\end{array}$ & $\begin{array}{l}\text { Last- } \\
\text { Image- } \\
\text { Hold }\end{array}$ & $\begin{array}{l}\text { Röntgen- } \\
\text { bilder }\end{array}$ & $\begin{array}{c}\text { Dosisflächen- } \\
\left.\text { Produkt [ } \mu G y^{*} m^{2}\right]\end{array}$ & $\begin{array}{c}\text { Augen- } \\
\text { dosis [mSv] }\end{array}$ \\
\hline 38 & 8 & 5 & 0 & 3,8 & 0,002 \\
\hline 5 & 9 & 5 & 0 & 4,6 & 0,018 \\
\hline 24 & 7 & 2 & 0 & 6,7 & 0,001 \\
\hline 1 & 7 & 3 & 0 & 11,5 & 0,006 \\
\hline 15 & 39 & 15 & 0 & 15,0 & $-0,003$ \\
\hline 35 & 25 & 7 & 0 & 21,5 & 0,006 \\
\hline 4 & 15 & 4 & 0 & 22,7 & 0,032 \\
\hline 2 & 12 & 3 & 0 & 32,3 & 0,016 \\
\hline 41 & 25 & 5 & 0 & 35,1 & 0,008 \\
\hline 37 & 14 & 4 & 0 & 36,2 & 0,012 \\
\hline 43 & 34 & 7 & 0 & 38,2 & $-0,003$ \\
\hline 18 & 23 & 4 & 1 & 42,4 & $-0,001$ \\
\hline 31 & 41 & 5 & 0 & 64,2 & 0,017 \\
\hline 42 & 56 & 6 & 0 & 68,9 & 0,006 \\
\hline 25 & 49 & 5 & 0 & 70,3 & 0,007 \\
\hline 30 & 34 & 5 & 0 & 74,0 & 0,013 \\
\hline 36 & 69 & 6 & 2 & 116,0 & 0,002 \\
\hline 26 & 58 & 4 & 0 & 255,8 & 0,018 \\
\hline 8 & 59 & 9 & 1 & 277,9 & 0,013 \\
\hline 6 & 58 & 9 & 0 & 355,9 & 0,003 \\
\hline 44 & 273 & 4 & 9 & 409,6 & 0,003 \\
\hline 12 & 234 & 8 & 4 & 487,8 & 0,001 \\
\hline Mittelwert & 52,2 & 5,7 & 0,8 & 111,4 & 0,008 \\
\hline
\end{tabular}


Wie auch bei den Messungen unter Standardbedingungen im Phantommodell stieg ohne Verwendung eines Strahlenschutzvisiers die Augenlinsendosis mit zunehmendem Dosisflächenprodukt an. Allerdings war die gemessene Augenlinsendosis unter den Realbedingungen einer ureterorenoskopischen Steinentfernung deutlichen Schwankungen zwischen -0,010 mSv und 0,018 mSv unterworfen, mit einem Mittelwert von 0,005 mSv bei einer Standardabweichung von $\pm 0,008 \mathrm{mSv}$. Der Anstieg der Augenlinsendosis war dabei besonders bei hohem Dosisflächenprodukt beobachtbar (Abb.14).

Auch bei den Messungen mit Strahlenschutzvisier wies die gemessene Augenlinsendosis deutliche Schwankungen zwischen $-0,003$ und 0,032 mSv auf, bei einem Mittelwert von 0,008 mSv und einer Standardabweichung von $\pm 0,008$ mSv. Auffallend war hier der fehlende Anstieg bei zunehmendem Dosisflächenprodukt (Abb.15).

Beim Vergleich aller Untersuchungen ohne Strahlenschutzvisier und unter Verwendung eines Strahlenschutzvisiers finden sich keine statistischen Unterschiede bei der akquirierten Augenlinsendosis (Tab.13).

Im Trend bestätigt sich die Erhöhung der Augenlinsendosis mit zunehmender Strahlenexposition (gemessen am Dosisflächenprodukt) ohne Strahlenschutzvisier, wohingegen es bei Verwendung eines Strahlenschutzvisiers im Trend zu keiner wesentlichen Zunahme der Augenlinsendosis bei zunehmendem Dosisflächenprodukt kommt (Abb.16). Allerdings können auch hier bei der getrennten Betrachtung der ureterorenoskopischen Eingriffe mit einem Dosisflächenprodukt von unter $50 \mu \mathrm{Gy}^{*} \mathrm{~m}^{2}$ und den Eingriffen mit einem Dosisflächenprodukt von über $50 \mu \mathrm{Gy}^{*} \mathrm{~m}^{2}$ keine statistisch signifikanten Unterschiede in der Augendosisbelastung unter den Realbedingungen ohne bzw. mit Verwendung eines Strahlenschutzvisiers gefunden werden $(p=0,1159$ bzw. $p$ $=0,1982$ ) (Abb.17 und Abb.18). 
Abbildung 14

Verhältnis von Dosisflächenprodukt und Augenlinsendosis bei zunehmendem Dosisflächenprodukt ohne Strahlenschutzvisier

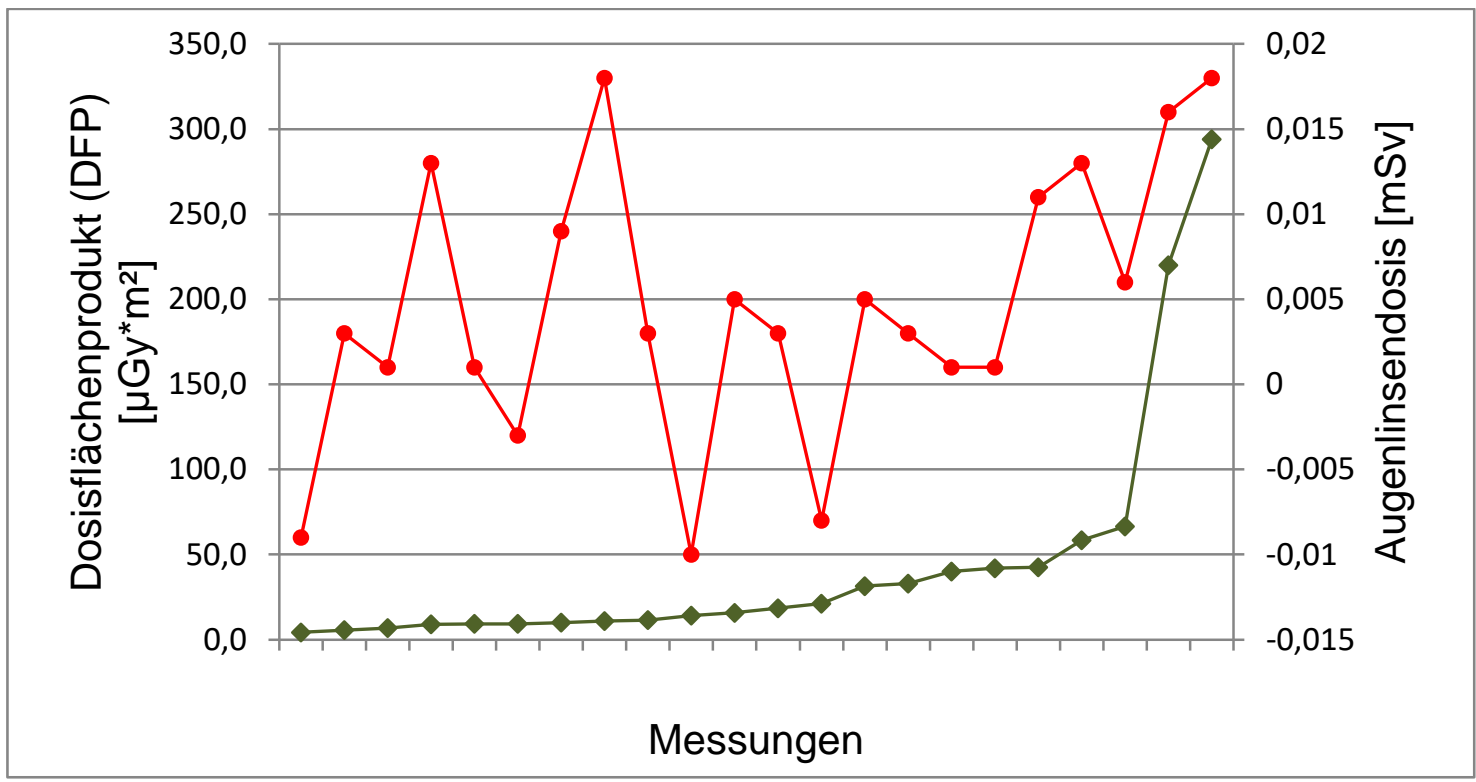

\section{Abbildung 15}

Verhältnis von Dosisflächenprodukt und Augenlinsendosis bei zunehmendem Dosisflächenprodukt mit Strahlenschutzvisier

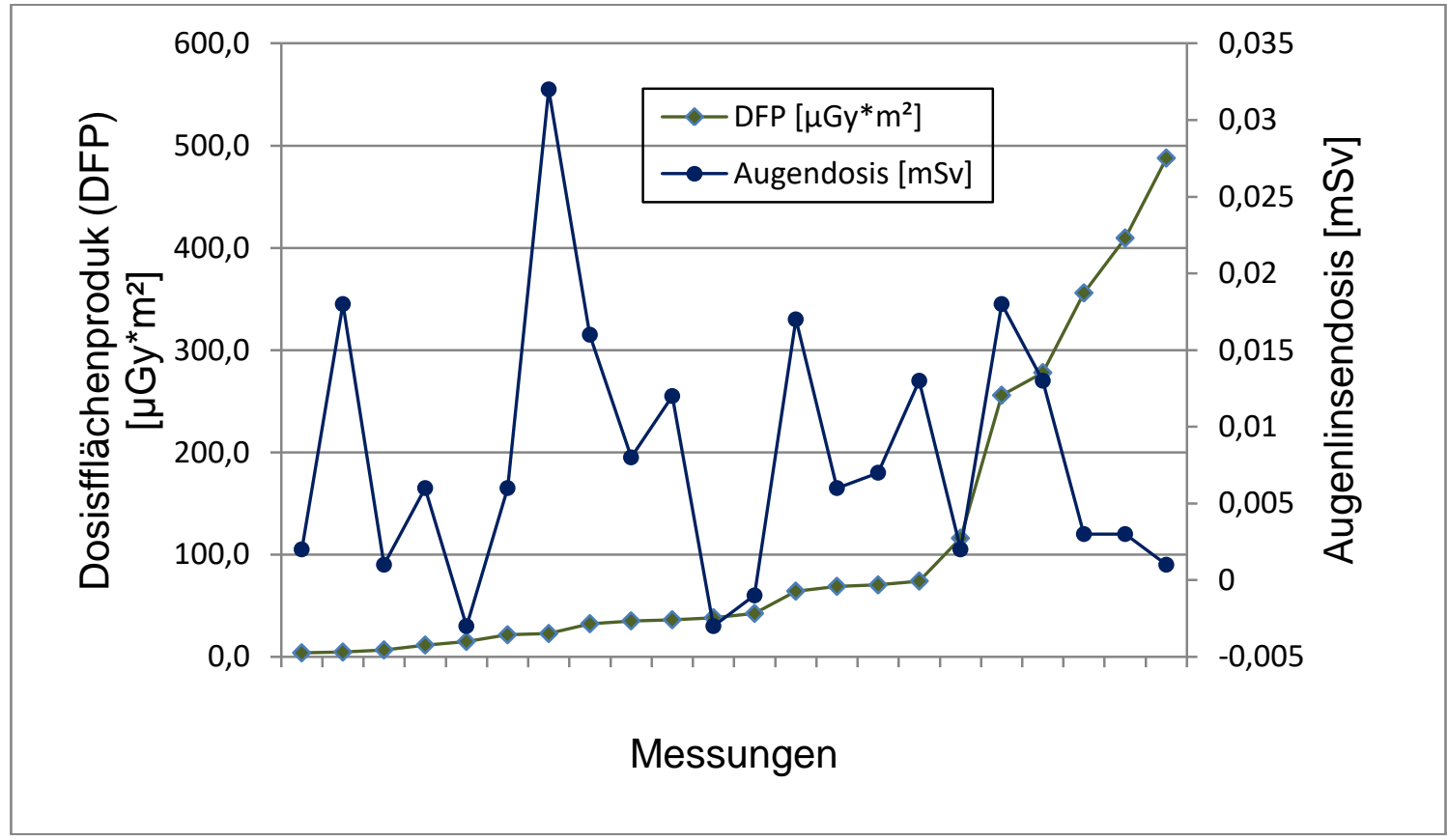




\section{$\underline{\text { Tabelle } 13}$}

Vergleich der Messungen ohne und mit Strahlenschutzvisier

\begin{tabular}{|c|c|c|c|}
\hline & $\begin{array}{c}\text { ohne Strahlen- } \\
\text { schutzvisier }\end{array}$ & $\begin{array}{l}\text { mit Strahlen- } \\
\text { schutzvisier }\end{array}$ & $\begin{array}{l}\text { Signifikanz- } \\
\text { niveau } \\
\text { (t-Test) }\end{array}$ \\
\hline Eingriffszahl & 22 & 22 & \\
\hline $\begin{array}{l}\text { Röntgenaufnahme } \\
\text { Mittelwert } \pm \\
\text { Standardabweichung }\end{array}$ & $0,1 \pm 0,4$ & $0,8 \pm 2$ & $p=0,1375$ \\
\hline $\begin{array}{l}\text { Durchleuchtungszeit } \\
\text { [Sekunden] } \\
\text { Mittelwert } \pm \\
\text { Standardabweichung }\end{array}$ & $27,7 \pm 32,5$ & $52,7 \pm 66,7$ & $p=0,1377$ \\
\hline $\begin{array}{l}\text { Dosisflächenprodukt } \\
{\left[\mu G{ }^{*} m^{2}\right]} \\
\text { Mittelwert } \pm \\
\text { Standardabweichung }\end{array}$ & $44,3 \pm 70,3$ & $111,4 \pm 141,9$ & $p=0,0580$ \\
\hline $\begin{array}{l}\text { Augenlinsendosis } \\
{[\mathrm{mSv}]} \\
\text { Mittelwert } \pm \\
\text { Standardabweichung }\end{array}$ & $0,005 \pm 0,008$ & $0,008 \pm 0,008$ & $p=0,1706$ \\
\hline
\end{tabular}


Abbildung 16

Verhältnis von Dosisflächenprodukt und Augenlinsendosis bei zunehmendem Dosisflächenprodukt ohne und mit Strahlenschutzvisier (mit Trendlinie in logarithmischer Skalierung = Log.)

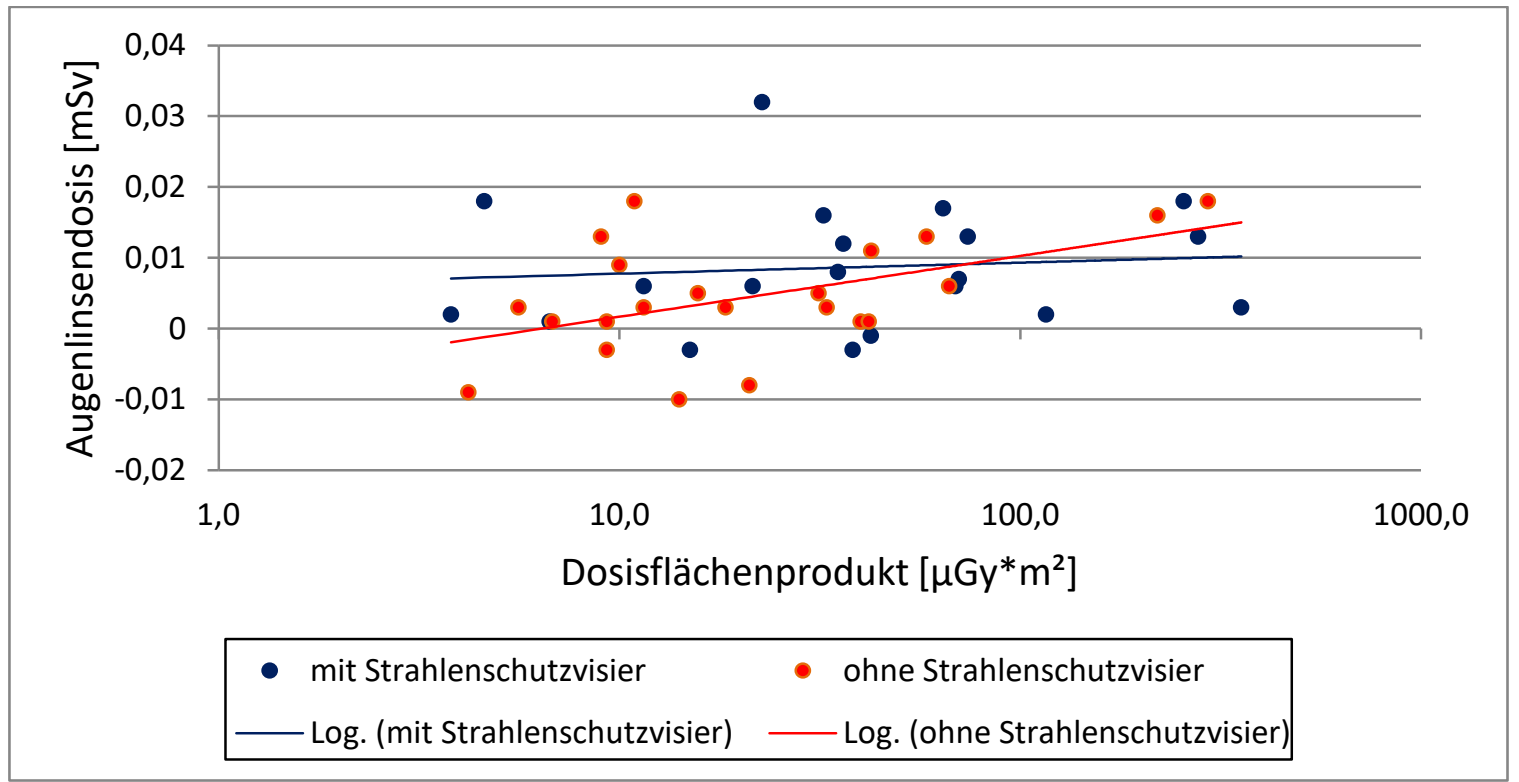


Abbildung 17

Augenlinsendosis bei Eingriffen mit einem Dosisflächenprodukt von jeweils unter $50 \mu \mathrm{Gy}^{\star} \mathrm{m}^{2}$ ohne und mit Verwendung eines Strahlenschutzvisiers $(p=0,1159, \mathrm{t}-$ Test)

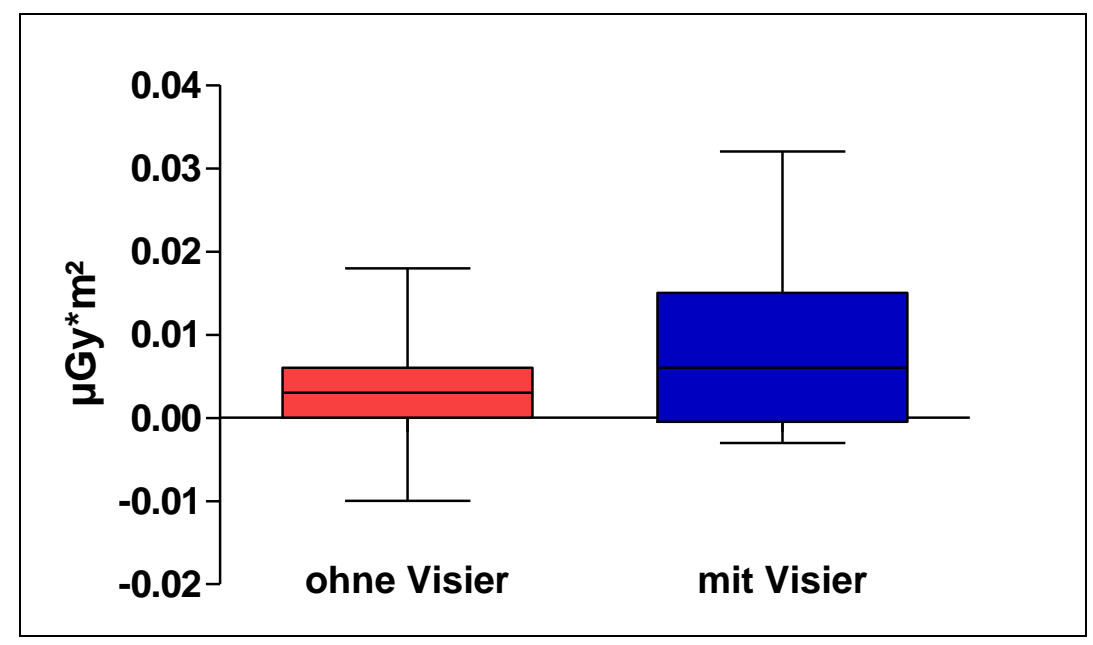

Abbildung 18

Augenlinsendosis bei Eingriffen mit einem Dosisflächenprodukt von jeweils über $50 \mu \mathrm{Gy}^{\star} \mathrm{m}^{2}$ ohne und mit Verwendung eines Strahlenschutzvisiers $(p=0,1982, t-$ Test)

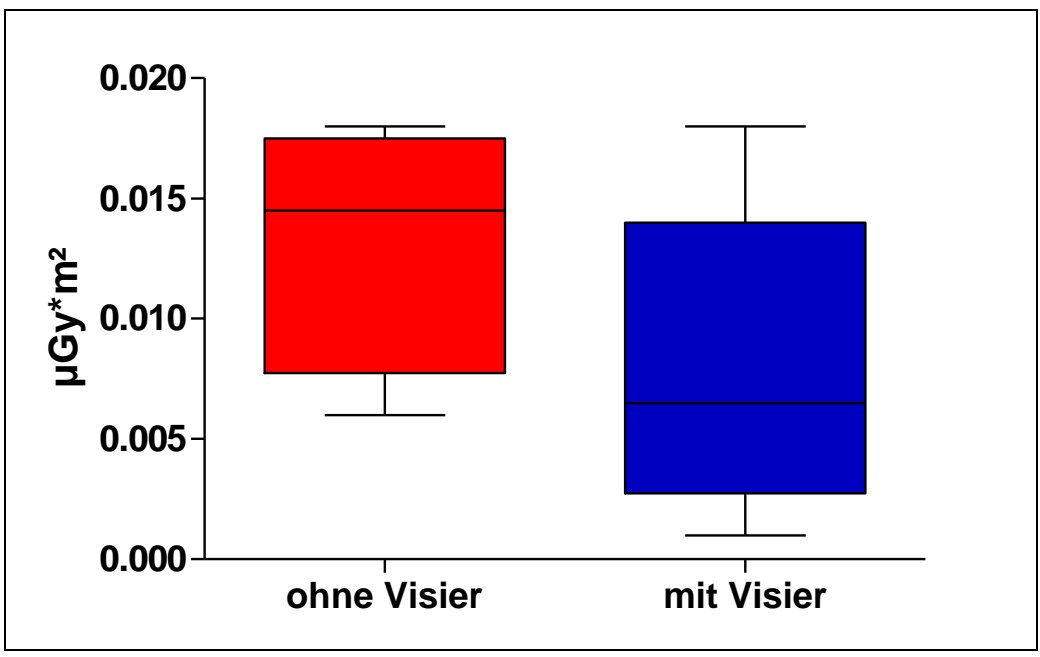




\section{Diskussion}

Die moderne Medizin ist gekennzeichnet durch die zunehmende Verbreitung minimal-invasiver Behandlungsmethoden, die invasive offen-chirurgische Behandlungsmethoden vermeiden hilft. Dies gilt bespielhaft für kardiovaskuläre Erkrankungen, für gastrointestinale Erkrankungen, aber auch für urologische Erkrankungen. Minimal-invasive endoskopische Interventionen bedürfen dabei in der Regel einer simultanen Bildgebung, häufig durch intraoperative Röntgenuntersuchungen. Die Vorteile der minimal-invasiven Behandlung werden deshalb mit einer zunehmenden Röntgenexposition „erkauft“. Diese Röntgenexposition betrifft sowohl den Patienten selbst als auch das Behandlungsteam, insbesondere den interventionell tätigen Arzt, der häufig nahe an der Röntgenquelle arbeiten muss.

Die Strahlenbelastung für Patienten ist hinreichend untersucht. Sie ist abhängig von der Komplexität der Intervention mit den sich daraus ergebenden Durchleuchtungszeiten.

Eine Übersichtsarbeit zur Strahlenbelastung bei interventionellen kardiologischen Prozeduren wurde von Picano et al. 2014 publiziert. Danach beträgt nach unterschiedlichen Publikationen die Strahlenbelastung für Patienten bei interventionellen kardiologischen Eingriffen wie der perkutanen koronaren Intervention oder für eine Radiofrequenzablation 1 bis $60 \mathrm{mSv}$, im Mittel ca. 15 mSv pro Eingriff (Picano et al. 2014).

In der Gastroenterologie liegen beispielhaft Daten zur Strahlenbelastung bei der endoskopischen retrograden Cholangiopankreatikographie (ERCP) vor. Dabei wurde für Patienten eine mittlere effektive Dosis von 4,16 mSv pro Eingriff nachgewiesen (Alzimami et al. 2013).

In der Urologie wurden Daten zur Strahlenbelastung der Patienten bei der extrakorporalen Stoßwellenlithotripsie, der perkutanen Nephrolitholapaxie und der ureterorenoskopischen Steinentfernung publiziert. Nach Untersuchungen von Sandilos et al. beträgt die mittlere effektive Dosis für Patienten bei der extrakorporalen Stoßwellenlithotripsie im Mittel 1,63 mSv (Sandilos et al. 2006). 
Für die perkutane Nephrolitholapaxie ermittelten vorangegangene Untersuchungen eine durchschnittliche Strahlenbelastung für Patienten von 0,56 (Kumari et al. 2006) bzw. in Abhängigkeit vom Body-Mass-Index von 2,66 bis 9,13 mSv pro Eingriff (Mancini et al. 2010).

Zur Strahlenbelastung für Patienten bei der ureterorenoskopischen Steinentfernung wurden im Jahr 2013 Daten aus unserer Klinik publiziert. Die Untersuchungen erfolgten ebenfalls unter Verwendung der urologischen Behandlungseinheit Uroskop Access ${ }^{\circledR}$, die in der hier vorgestellten Arbeit zum Einsatz kam. Dabei wurde zunächst durch die im Strahlengang befindliche Ionisationskammer das Dosisflächenprodukt gemessen und anschließend unter Berücksichtigung eines spezifischen Konversionsfaktors (0,00323 mSv / $\mu \mathrm{Gy} x$ $\mathrm{m}^{2}$ ) die effektive abdominelle Dosis berechnet. Diese belief sich auf durchschnittlich 0,67 mSv pro Eingriff bei der semirigiden Ureterorenoskopie, $1,17 \mathrm{mSv}$ pro Eingriff bei der flexiblen Ureterorenoskopie und 2,23 mSv pro Eingriff bei komplizierten Eingriffen mit simultaner Behandlung von Harnleiterund Nierensteinen. Die Strahlenbelastung ist dabei abhängig vom Aufwand des Eingriffs, insbesondere von der Steinlage, der Form der Ureterorenoskopie (semirigide oder flexibel) und der Notwendigkeit einer Laserlithotripsie. Das Dosisflächenprodukt korrelierte dabei erwartungsgemäß mit der intraoperativen Durchleuchtungszeit, während die intraoperativ ausgelösten Röntgenaufnahmen nur gering zur Dosisbelastung beitrugen. Dies liegt daran, dass zur Röntgendokumentation hauptsächlich Last-image-hold-Aufnahmen des letzten Durchleuchtungsbildes ohne zusätzliche Strahlenbelastung genutzt wurden. Zudem zeigte sich, dass die interindividuellen Präferenzen der Operateure hinsichtlich der intraoperativen radiologischen Kontrolle des endoskopischen Eingriffs zu unterschiedlichen Strahlenbelastungen der Patienten führten (Zöller et al. 2013).

Diese Untersuchungen zeigen, dass die intraoperative Strahlenbelastung von Patienten bei der ureterorenoskopischen Steinentfernung unter Verwendung moderner Röntgensysteme in der Regel deutlich unter der Strahlenbelastung bei interventionellen kardiologischen oder interventionellen gastroenterologischen Eingriffen liegt. 
Neben der Strahlenbelastung für den Patienten rückt in den letzten Jahren zunehmend die Strahlenbelastung des interventionell tätigen Arztes immer mehr in den Fokus, da hier trotz der persönlichen Schutzmaßnahmen wie Röntgenschürze oder Schilddrüsenschutz die kumulative, im Lauf des Berufslebens erworbene Strahlenbelastung erheblich sein kann.

Auch die Frage nach der Strahlenbelastung des interventionell tätigen Arztes wurde auf Grund der bekannten erheblichen Strahlenbelastung für Patienten insbesondere im Bereich der interventionellen Kardiologie untersucht. Zusammenfassende Ergebnisse sind in den Arbeiten von Martin (2009), Kesavachandran et al. (2013) oder Badawy et al. (2016) dargestellt. Pro Eingriff wurde eine effektive Dosis von 0,0002 bis 0,004 mSv errechnet; vereinzelt wurde jedoch auch über Strahlenbelastungen von bis zu 0,019 mSv pro Eingriff berichtet. Danach beträgt die kumulative Strahlenbelastung in dieser besonders exponierten Berufsgruppe bei 500 Eingriffen pro Jahr durchschnittlich 0,1 - 2 mSv. Aber auch in anderen Fachdisziplinen wie der Gastroenterologie ist die Problematik erkannt. Hier publizierten Seo et al. (2016) Daten zur Strahlenbelastung des medizinischen Personals bei 126 Cholangiopankreatikographien $(E R C P)$. Die Untersuchungen erfolgten unter Verwendung von Thermolumineszenz-Dosimetern. Dabei wurde für den Operateur eine durchschnittliche effektive Dosis von 0,175 mSv pro Eingriff ermittelt.

Im urologischen Fachgebiet sind dagegen kaum Daten zur Strahlenbelastung des Operateurs bei minimal-invasiven interventionellen Eingriffen publiziert.

In einer Vergleichsstudie von Vano et al. zeigte sich bei den 34 durchgeführten urologischen Interventionen (Nephrolithotomien) eine 18,7mal höhere Strahlenbelastung des Operateurs als bei interventionell tätigen Radiologen und Kardiologen und eine 4,2mal höhere Strahlenbelastung als bei Gefäßchirurgen der gleichen Klinik. Die mediane, allerdings im experimentellen Ansatz erstaunlicherweise über der Bleischürze gemessene Äquivalentdosis belief sich dabei auf 0,393 mSv pro urologischem Eingriff beim Operateur und 0,021 mSv pro Eingriff beim instrumentierenden Assistenzpersonal (Vano et al. 2013). 
Eine relativ neue Studie aus Frankreich beschäftigte sich mit der Strahlenbelastung von 12 interventionell tätigen urologischen Operateuren, sowie mit ihrem Bewusstsein für Strahlenschutzmaßnahmen (Galonnier et al. 2016). Dabei wurde eine jährliche Ganzkörper-Äquivalentdosis von 0,1 - 0,8 mSv pro Operateur erreicht. Die durchgeführten Strahlenschutzmaßnahmen waren alarmierend schlecht - nur 15\% der Urologen trugen überhaupt ein Dosimeter und nur 5\% waren in den Umgang mit der Röntgenanlage eingewiesen worden. Diese Daten aus Frankreich lassen sich damit nicht ohne weiteres auf Deutschland übertragen, da hier Strahlenschutzkurse und jährliche Unterweisungen verpflichtend sind.

Von besonderem Interesse bei der Strahlenbelastung des behandelnden Arztes ist dabei die Augenlinsendosis, da die Augenlinse des Operateurs als strahlensensibles Gewebe durch ihre operationsbedingte räumliche Nähe zur Röntgenröhre besonders gefährdet ist. Die Entwicklung einer strahlenbedingten Katarakt ist in Studien am Tiermodell und an Menschen nachgewiesen worden. Mit zunehmender Strahlenbelastung steigt dabei die Schädigung des Gewebes und somit die Wahrscheinlichkeit und Schwere der Erkrankung (deterministische Strahlenwirkung).

Auch für die Strahlenbelastung der Augenlinsen und das Kataraktrisiko gibt es im Bereich der interventionellen Kardiologie die meisten Voruntersuchungen. (Antic et al. 2013, Principi et al. 2015, Sanchez et al. 2016a und 2016b, Domienik et al. 2016). Dabei wurde der Frage der Strahlenbelastung sowohl hinsichtlich der Augenlinsendosis für einzelne Untersuchungen mit und ohne besondere Strahlenschutzmaßnahmen wie auch der Frage der kumulativen Augenlinsendosis während eines Berufslebens nachgegangen. Zudem existieren epidemiologische Daten zur strahleninduzierten Kataraktinzidenz für interventionell tätige Kardiologen.

Die Strahlenbelastung der Augen bei interventionellen kardiologischen Eingriffen beträgt in diesen Untersuchungen im Mittelwert 0,046 mSv pro Eingriff, wobei Eingriffe bei Herzklappen- und anderen strukturellen Herzerkrankungen sogar auf Mittelwerte über 0,1 mSv pro Prozedur kommen (Sanchez et al. 2016). 
Antic et al. (2013) wiesen eine Augenlinsendosis von 0,121 mSv pro Eingriff für den Operateur nach. Principi et al. (2015) errechneten eine Strahlenbelastung von 8 bis 60 mSv bei einer Durchführung von 200 Eingriffen pro Jahr. Andere Studien geben eine kumulative Augenlinsendosis bis zu 1,55 Sv jährlich an (Domienik et al. 2016b).

Untersuchungsergebnisse aus der Gastroenterologie zeigten, dass die Augenlinsendosis in Abhängigkeit von der Röntgenanlage mit bis zu 0,09 mSv pro ERCP bei häufigen Eingriffen die empfohlene jährliche Höchstdosis von 20 mSv überschreiten kann (O'Connor et al. 2013). Dies bestätigten auch die Untersuchungen von Zagorska et al. (2015), die eine durchschnittliche Augenlinsendosis von 0,0349 bis 0,0933 mSv pro Untersuchung errechneten.

Vergleichbare Untersuchungen in der Urologie wurden bisher nur dreimal publiziert (Ritter et al. 2012, Hristova-Popova et al. 2015, Galonnier et al. 2016). Ritter et al. haben in einer prospektiven Single-center-Studie die Strahlenbelastung des Operateurs bei insgesamt 235 endourologischen Eingriffen im Zeitraum von April bis September 2010 untersucht. Die Eingriffe umfassten neben Ureterorenoskopien auch perkutane Nephrolitholapaxien, DJ-Schienenwechsel und perkutane Stentwechsel. Bei den Eingriffen trug der Operateur jeweils zwei Thermolumineszenz-Dosimeter - eines im Stirnbereich zur Messung der Augenlinsen- sowie der Schilddrüsen-Dosis, und eines am Ringfinger derjenigen Hand, die sich näher am Strahlengang befand. Als Strahlenquelle diente eine Over-the-table-Röntgenanlage, wie sie zumeist in der Urologie Verwendung findet. Die Operateure trugen Bleischürzen sowie Schilddrüsenschutz und eine Strahlenschutzbrille. Sie wurden je nach ihrer Erfahrung in der Endourologie (unter bzw. über 2 Jahre) in zwei Gruppen eingeteilt. Die Dosimeter wurden alle 3 Monate im Helmholtz-Institut in München ausgewertet. Aus diesen Kumulativwerten wurden anschließend die Durchschnittswerte für die einzelnen Eingriffe berechnet. Insgesamt wurden in dieser Studie 39 Ureterorenoskopien durchgeführt; dabei wurde eine durchschnittliche Durchleuchtungsdauer von 1,1 Minuten (Minimum 0,2, Maximum 13,9 Minuten) angegeben. 
Dabei konnten eine durchschnittliche Strahlenbelastung der Augenlinse des Operateurs von 0,1 mSv für die Ureterorenoskopie und 0,18 mSv für eine perkutane Nephrolitholapaxie errechnet werden.

Hristova-Popova et al. untersuchten zunächst am Phantommodell und dann während tatsächlich durchgeführter Eingriffe (15 Ureterorenoskopien und 16 perkutane Nephrolithotomien) die Augenlinsendosis mit und ohne Bleischirm als Strahlenschutzmaßnahme. Die durchgeführten Eingriffe wurden immer vom selben, erfahrenen Endourologen durchgeführt. Das Dosimeter wurde dabei zwischen den Augen platziert und einen Monat lang getragen. Für ureterorenoskopische Eingriffe wiesen Hristova-Popova et al. eine kumulative Augenlinsendosis von 0,9 mSv ohne Strahlenschutzmaßnahmen und 0,06 mSv unter Verwendung eines Bleischirms nach. Dabei lag die durchschnittliche Durchleuchtungszeit bei 0,9 Minuten (0,1 bis 3,4 Minuten); es wurden durchschnittlich 2 Röntgenbilder (0 bis 10 Röntgenaufnahmen) angefertigt. Für perkutane Interventionen lag die Augenlinsendosis sogar bei durchschnittlich 1,9 mSv ohne und bei durchschnittlich 0,02 mSv mit Bleischutzschirm. Die durchschnittliche Durchleuchtungszeit lag hier bei 4,5 Minuten, es wurden zusätzlich 6 Röntgenaufnahmen ausgelöst. Die jährliche Augenlinsendosis des Operateurs bei üblicher Arbeitsbelastung wurde daher auf $29 \mathrm{mSv}$ ohne die Verwendung eines Strahlenschutzschirms und 0,9 mSv unter Verwendung eines Strahlenschutzschildes geschätzt. Dabei wurde von 200 Arbeitstagen im Jahr und einer perkutanen Nephrolithotomie täglich ausgegangen.

Galonnier et al. führten eine bizentrische, prospektive Studie durch. Dabei erfolgten die Messungen einen Monat lang an 12 Operateuren während endourologischer Operationen sowie am Phantom. Die operativen Eingriffe umfassten retrograde Einlagen von Harnleiterschienen (DJ-Schienen), Ureterorenoskopien und perkutane Nephrolithotomien. Die Operateure trugen sieben Dosimeter am Körper, u. a. an der Stirn. Am antropomorphen Phantom anstelle des Patienten erfolgten zusätzliche Untersuchungen mit prolongierten Bestrahlungszeiten von 6 Minuten. Die Studie wurde in einem Zeitintervall von 15 Tagen in den beiden Zentren durchgeführt; dabei wurden 35 Ureterorenoskopien durchgeführt. 
11 der 12 Operateure trugen ihre Dosimeter korrekt, einer verzichtete vollständig auf die Dosimeter, und zwei Dosimeter gingen verloren. Für die einzelne Ureterorenoskopie konnte bei 8 der 11 Operateure, die Dosimeter trugen, keine Strahlendosis gemessen werden bzw. lag die Strahlendosis unter der Erkennungsgrenze von $10 \mu \mathrm{Sv}$. Zur Einschätzung der kumulativen jährlichen Strahlendosis wurden dann die durchschnittlichen Operationszahlen der Klinik zugrunde gelegt, die bei 86 Ureterorenoskopien, 8 perkutanen Nephrolitholapaxie und 28 retrograden DJ-Einlagen pro Operateur und Jahr lagen. Die Berechnung der Kumulativdosis erfolgte anhand von „URSäquivalenten Dosen“ (3 retrograde DJ-Einlagen $=1$ Ureterorenoskopie $=0,5$ perkutane Nephrolitholapaxie). Hieraus ergab sich eine errechnete jährliche Augenlinsendosis von 0,31-2,3 mSv (Galonnier et al. 2016).

Daten zur Dosisbelastung der Augenlinse des Operateurs sind damit für die interventionellen endourologischen Eingriffe nur spärlich vorhanden und aufgrund von Unterschieden im operativen Spektrum und in den angewandten Strahlenschutzmaßnahmen nicht auf unsere Klinik übertragbar.

Wir sind deshalb in der vorgelegten Arbeit der Strahlenbelastung der Augenlinse des Operateurs bei der wichtigsten bei uns durchgeführten interventionellen endourologischen Operation, der ureterorenoskopischen Steinentfernung, nachgegangen.

Wir haben dabei in einem ersten experimentellen Schritt in einem Phantommodell die Augenlinsendosis in Abhängigkeit von der Durchleuchtungszeit und der Anzahl der Röntgenaufnahmen bestimmt. Zur Simulation des Operateurs wurde ein Kopfmodell mit daran angebrachtem Augendosimeter in typischer Entfernung zur Röntgenanlage platziert. Zur Erzeugung einer realitätsentsprechenden Streustrahlung erfolgten die Untersuchungen unter Verwendung eines Alderson-Phantoms, das auf dem Röntgentisch platziert wurde. Anschließend wurden 1 - 10 Röntgenaufnahmen angefertigt, das Dosimeter dazwischen jeweils gewechselt. In einer weiteren Untersuchung wurden Messungen mit ansteigender Durchleuchtungszeit durchgeführt. 
Die Durchleuchtungszeit wurde in 15-Sekunden-Intervallen gesteigert und dafür jeweils ein neues Augendosimeter verwendet. Die Messungen erfolgten sowohl ohne als auch mit Verwendung eines Strahlenschutzvisiers.

Ohne Strahlenschutzvisier wurde dabei eine Zunahme der Strahlendosis im Augendosimeter von durchschnittlich 0,002 mSv bei einer Röntgenaufnahme auf durchschnittlich $0,008 \mathrm{mSv}$ bei 10 Röntgenaufnahmen sowie von 0,002 mSv bei 15 Sekunden Durchleuchtungszeit auf 0,024 mSv bei 180 Sekunden Durchleuchtungszeit gemessen.

Mit Strahlenschutzvisier betrug die unter vergleichbaren Bedingungen gemessene Strahlendosis im Augendosimeter durchschnittlich 0,002 mSv bei einer Röntgenaufnahme und durchschnittlich $-0,001 \mathrm{mSv}$ bei 10 Röntgenaufnahmen sowie durchschnittlich 0,001 mSv bei einer Durchleuchtungszeit von 15 Sekunden und durchschnittlich 0,000 mSv bei einer Durchleuchtungszeit von 180 Sekunden.

Negative Werte ergeben sich dabei rechnerisch durch die Subtraktion der Hintergrundstrahlung, wie sie in unserem Untersuchungsaufbau vorgesehen war. Die Hintergrundstrahlung wurde in den wenigen vergleichbaren publizierten Studien unseres Wissens bisher nicht berücksichtigt.

Damit konnte in unseren Untersuchungen am Phantommodell durch die Verwendung des Strahlenschutzvisiers eine effektive Reduktion der Strahlendosis erreicht werden, insbesondere bei zunehmender Strahlenexposition durch eine höhere Anzahl von ausgelösten Röntgenaufnahmen oder bei zunehmender Durchleuchtungszeit. Aufgrund der geringen Strahlungsdosen und der großen Schwankungsbreite der gemessenen Strahlenbelastung wurde ein statistisches Signifikanzniveau $(<0,05, \mathrm{t}$-Test) allerdings nur in 2 von 10 Untersuchungsreihen mit zunehmender Zahl an Röntgenaufnahmen erreicht. Beim Vergleich der Strahlenbelastung bei ansteigender Durchleuchtungszeit zeigten die Untersuchungen mit einer Ausnahme eine statistisch signifikante Reduktion der Strahlenbelastung bei Verwendung eines Strahlenschutzvisiers ab einer Durchleuchtungszeit von 30 und mehr Sekunden. Diese Teilergebnisse der vorgelegten Arbeit wurden von uns 2015 publiziert (Zöller et al. 2015). 
Strahlenschutzbrillen und Strahlenschutzvisiere kommen im Bereich der interventionellen Kardiologie regelhaft zum Einsatz. Hier existieren auch Untersuchungen unter realistischen Alltagsbedingungen. Dabei konnte in einer klinischen Studie die Strahlendosis durch den Einsatz von Strahlenschutzvisieren bzw. -brillen um den Faktor 1,1 bis 3,4 und durch den Einsatz eines Strahlenschutzschildes um den Faktor 2,3 gesenkt werden (Domienik et al. 2016a).

Für endourologische Eingriffe wurde der Effekt einer Strahlenschutzbrille auf die Augenlinsendosis in der bereits zitierten Arbeit von Hristova-Popova et al. (2015) beschrieben. Für endourologische Eingriffe konnte dabei der Augenlinsendosis im Mittel von 0,9 mSv auf 0,06 mSv, speziell während perkutaner Interventionen von 1,9 auf 0,02 mSv gesenkt werden (HristovaPopova et al. 2015).

Allerdings handelt es sich hierbei um die über einen Monat akkumulierte Augenlinsendosis. Einzelmessungen für einzelne operative Eingriffe konnten aufgrund der Untersuchungsbedingungen mit einem monatlichen Auslesen der Augendosimeter nicht ermittelt werden. Auch hier blieb die Hintergrundstrahlung unberücksichtigt.

Wir haben deshalb in einem weiteren Schritt prospektiv randomisiert untersucht, ob durch die Verwendung von Strahlenschutzvisieren eine Reduktion der Strahlenbelastung am Auge des Operateurs unter den Realbedingungen einer ureterorenoskopischen Steinentfernung erreicht werden kann. Hierfür trug die Operateurin bei 45 ureterorenoskopischen Steinsanierungen ein Augendosimeter, von denen 22 ohne Strahlenschutzvisier und 23 mit Strahlenschutzvisier erfolgten. Aufgrund eines defekten Augendosimeters war einer der Eingriffe mit Strahlenschutzvisier nicht auswertbar, sodass letztlich jeweils 22 Augendosismeter, insgesamt 44 Augendosismeter für 44 operative Eingriffe, ausgewertet werden konnten.

Bei den 22 Eingriffen ohne Strahlenschutzvisier wurde eine Strahlendosis von 4,2 - 294,0 $\mu \mathrm{Gy}^{*} \mathrm{~m}^{2}$ (Mittelwert 44,3 $\mu \mathrm{Gy}^{*} \mathrm{~m}^{2}$ ) pro Eingriff nachgewiesen (gemessen als Dosis-Flächen-Produkt im Strahlengang). Bei den 22 Eingriffen 
unter Verwendung eines Strahlenschutzvisiers betrug die Strahlenbelastung 3,8 bis $487,8 \mu G y^{*} m^{2}$ (Mittelwert 111,4 $\mu \mathrm{Gy}^{*} \mathrm{~m}^{2}$ ) pro Eingriff.

Die Unterschiede in der mittleren Strahlenbelastung erklären sich retrospektiv durch einen höheren Schwierigkeitsgrad und die damit verbundene höhere Durchleuchtungszeit bei Eingriffen mit Strahlenschutzvisier. So betrug die Durchleuchtungszeit bei Eingriffen ohne Strahlenschutzvisier 7 bis 123 Sekunden (Mittelwert 27,7 Sekunden), mit Visier dagegen 7 bis 273 Sekunden (Mittelwert 52,2 Sekunden). Die unter den Realbedingungen einer ureterorenoskopischen Steinentfernung gemessene Augenlinsendosis wies aber deutliche Schwankungen auf und betrug ohne Strahlenschutzvisier zwischen -0,010 mSv und 0,018 mSv (Mittelwert 0,005 mSv). Mit Strahlenschutzvisier wurden Augenlinsendosen von -0,003 bis 0,032 mSv (Mittelwert 0,008 mSv) ermittelt. Während ohne Strahlenschutzvisier die Strahlenbelastung der Augenlinse mit zunehmendem Dosisflächenprodukt anstieg, fehlte dieser Anstieg bei der Verwendung eines Strahlenschutzvisiers. Die Unterschiede zwischen der Augenlinsenbelastung ohne und mit Strahlenschutzvisier waren in unserer Untersuchung aber statistisch nicht signifikant. Damit stehen unsere Ergebnisse im Gegensatz zur Arbeit von Hristova-Popova et al. 2015, wobei hier aber wie erwähnt eine kumulative Monatsdosis zur Berechnung der Strahlendosis herangezogen wurde.

Die Interpretation unserer Ergebnisse wird dabei durch die mangelnde Sensitivität des Augendosimeters für Einzelmessungen erschwert. Die Streubreite der Messergebnisse im Phantommodell ist insbesondere unter Berücksichtigung der natürlichen Hintergrundstrahlung (Nullwert) erheblich. Bei den Messungen unter Realbedingungen kam zudem durch die randomisierte Zuordnung der Eingriffe mit bzw. ohne Visier eine ungleiche Verteilung der Schwierigkeitsgrade und damit der Strahlenbelastung hinzu; in der Gruppe mit Strahlenschutzvisier befanden sich mehrere deutlich längere und schwierigere Eingriffe mit entsprechend längerer Durchleuchtungszeit und somit auch höherer Strahlenbelastung.

Unabhängig von den Messergebnissen fiel während der Eingriffe mit Verwendung des Strahlenschutzvisiers auf, dass durch die Schwere des Visiers 
und die ungleichmäßige Verteilung des Gewichts insbesondere bei längerdauernden Eingriffen regelmäßig Nacken- und Kopfschmerzen auftraten. Gerade bei diesen langdauernden Eingriffen, bei denen die Senkung der Strahlendosis durch das Visier am deutlichsten ausfällt, könnte damit aufgrund der Unhandlichkeit und Schwere des Strahlenschutzvisiers die Compliance des Operateurs beeinträchtigt sein und langfristige orthopädische Probleme mit entsprechender Behandlungsbedürftigkeit könnten auftreten.

Diese Probleme können durch die Verwendung von deutlich leichteren Strahlenschutzbrillen statt Strahlenschutzvisieren weitgehend umgangen werden. Allerdings stehen Strahlenschutzbrillen für Brillenträger nur mit begrenzter Sehstärkenkorrektur zur Verfügung (zum Beispiel von AIBO Medizintechnik: Sphäre +6 bis -9 Dioptrien oder von Safety First Deutschland: Sphäre $+3,5$ bis $-3,5$ Dioptrien). Zudem können mit den aktuell verfügbaren Augendosimetern Vergleichsuntersuchungen für ureterorenoskopische Eingriffe mit und ohne Strahlenschutzbrillen unter Realbedingungen nicht durchgeführt werden.

Die Entwicklung miniaturisierter Augendosimeter mit hoher Empfindlichkeit ist für weitere Untersuchungen zur Strahlenbelastung der Augenlinsen bei einzelnen interventionellen Eingriffen notwendig. 


\section{Zusammenfassung}

Interventionell tätige Urologen führen heute eine Vielzahl urologischer Eingriffe endoskopisch unter Bildwandlerkontrolle durch, zum Beispiel die ureterorenoskopische Steinsanierung. Auch wenn sie sich dabei nicht im direkten Strahlengang befinden, so erreicht sie dennoch ein gewisser Prozentsatz an Streustrahlung. Dabei ist insbesondere die Augenlinse als strahlensensibles Organ gefährdet, und es kann sich eine strahleninduzierte Katarakt entwickeln. Hierbei handelt es sich um eine deterministische Strahlenwirkung, bei der nach Überschreiten eines Schwellenwertes die Erkrankung eintritt und mit zunehmender Dosissteigerung auch der Schweregrad der Erkrankung zunimmt. Während die Strahlenbelastung der Patienten während endourologischer Eingriffe gut erforscht ist, gibt es nur eine unzureichende Datenlage zur Strahlenbelastung der Augenlinse des interventionell tätigen Urologen.

Daher haben wir zunächst in einem Phantommodell die Strahlenbelastung in Abhängigkeit von der Durchleuchtungszeit und der Anzahl der angefertigten Röntgenbilder ohne und mit Strahlenschutzvisier untersucht. Ohne Strahlenschutzvisier nahm die Strahlendosis im Augendosimeter von durchschnittlich 0,002 mSv bei einer Röntgenaufnahme auf durchschnittlich $0,008 \mathrm{mSv}$ bei 10 Röntgenaufnahmen zu sowie von durchschnittlich 0,002 mSv bei 15 Sekunden auf durchschnittlich $0,024 \mathrm{mSv}$ bei 180 Sekunden Durchleuchtungszeit. Mit Strahlenschutzvisier betrug die Strahlendosis im Augendosimeter durchschnittlich 0,002 mSv bei einer Röntgenaufnahme und durchschnittlich -0,001 mSv bei 10 Röntgenaufnahmen sowie durchschnittlich 0,001 mSv bei einer Durchleuchtungszeit von 15 Sekunden und durchschnittlich 0,000 mSv bei einer Durchleuchtungszeit von 180 Sekunden. Im Vergleich der Untersuchungsreihen konnte die gemessene Strahlendosis am Augendosimeter durch die Verwendung eines Strahlenschutzvisiers bei zunehmendem Dosisflächenprodukt, insbesondere bei zunehmender Durchleuchtungszeit, deutlich gesenkt werden.

Anschließend wurde die Augenlinsendosis während 45 ureterorenoskopischen Eingriffen mit und ohne Strahlenschutzvisier gemessen. 
Davon waren letztlich 22 Eingriffe mit Strahlenschutzvisier und 22 Eingriffe ohne Strahlenschutzvisier auswertbar. Die unter den Realbedingungen ermittelte Augenlinsendosis betrug ohne Strahlenschutzvisier zwischen -0,010 $\mathrm{mSv}$ und 0,018 mSv (Mittelwert 0,005 mSv), mit Strahlenschutzvisier -0,003 bis 0,032 mSv (Mittelwert 0,008 mSv). Ohne Strahlenschutzvisier stieg die Strahlenbelastung der Augenlinse mit zunehmendem Dosisflächenprodukt an, während ein solcher Anstieg bei der Verwendung eines Strahlenschutzvisiers nicht beobachtet wurde. Die Unterschiede zwischen der Augenlinsenbelastung ohne und mit Strahlenschutzvisier waren aber in unserer Untersuchung statistisch nicht signifikant.

Während sich damit im experimentellen Teil mit Zunahme der Durchleuchtungszeit auch eine Zunahme der Augenlinsendosis zeigte und ab einer Durchleuchtungszeit von 30 Sekunden durch die Verwendung eines Strahlenschutzvisiers auch eine statistisch signifikante Reduktion der Augenlinsendosis nachgewiesen werden konnte, wurde unter Realbedingungen das Signifikanzniveau für die Einzelmessungen nicht erreicht. Dennoch legen die Untersuchungen nahe, dass insbesondere bei Operateuren, die häufig endourologische Eingriffe unter Bildwandlerkontrolle durchführen, insbesondere solche mit langen Durchleuchtungszeiten, ein Schutz der Augenlinsen mittels Strahlenschutzvisier bzw. -brille sinnvoll ist, um das Risiko einer Strahlenkatarakt zu minimieren. 


\section{Literaturverzeichnis}

Alzimami K, Sulieman A, Paroutoglou P, Potamianos S, Vlychou M, Theodorou K (2013): Optimisation of radiation exposure to gastroenterologists and patients during therapeutic ERCP. Gastroenterol Res Pract, 587574

Antic V, Ciraj-Bjelac O, Rehani M, Aleksandric S, Arandjic D, Ostojic M (2013): Eye lens dosimetry in interventional cardiology: results of staff dose measurements and link to patient dose levels. Radiat Prot Dosimetry 154, 27684

Badawy MK, Deb P, Chan R, Farouque O (2016): A review of radiation protection solutions for the staff in the cardiac catheterisation laboratory. Heart Lung Circ 25, 961-967

Chodick G, Bekiroglu N, Hauptmann M, Alexander BH, Freedman DM, Doody MM, Cheung LC, Simon SL, Weinstock RM, Bouville A, Sigurdson AJ (2008): Risk of cataract after exposure to low doses of ionizing radiation: a 20-year prospective cohort study among US radiologic technologists. Am J Epidemiol $\underline{168}, 620-31$

Domienik J, Bissinger A, Grabowicz W, Jankowski Ł, Kręcki R, Makowski M, Masiarek K, Plewka M, Lubiński A, Peruga JZ (2016a): The impact of various protective tools on the dose reduction in the eye lens in an interventional cardiology-clinical study. J Radiol Prot $\underline{36}, 309-318$

Domienik J, Gryglak S, Jurewicz J (2016b): Characteristics of interventional cardiologists and their work practices for the study on radiation-induced lens opacities based on the methodology developed by ELDO-preliminary results. $J$ Radiat Res $\underline{57}$, 431-437 
European Society of Radiology (2015): Summary of the European directive 2013/59/Euratom: essentials for health professionals in radiology. Insights Imaging $\underline{6}, 411-417$

Ferrufino-Ponce ZK, Henderson BA (2006): Radiotherapy and cataract formation. Semin Ophthalmol 21, 171-180

Galonnier F, Traxer O, Rosec M, Terrasa JB, Gouezel P, Celier D, Bassinet C, Ruffion A, Paparel P, Fiard G (2016): Surgical staff radiation protection during fluoroscopy-guided urologic interventions. J Endourol 30, 638-43

Hahn GA: Kurzlehrbuch Augenheilkunde. Thieme, Stuttgart 2012

Hassan M, El-Nahas AR, Sheir KZ, El-Tabey NA, El-Assmy AM, Elshal AM, Shokeir AA (2015): Percutaneous nephrolithotomy vs. extracorporeal shockwave lithotripsy for treating a $20-30 \mathrm{~mm}$ single renal pelvic stone. Arab $\mathrm{J}$ Urol $\underline{13}, 212-216$

Hesse A, Brändle E, Wilbert D, Köhrmann K-U, Alken P (2003): Study on the prevalence and incidence of urolithiasis in Germany comparing the years 1979 vs. 2000. Eur Urol $\underline{44}, 709-713$

Hristova-Popova J, Zagorska A, Saltirov I, Petkova K, Vassileva J (2015): Risk of radiation exposure to medical staff involved in interventional endourology. Radiat Prot Dosimetry $\underline{165}$, 268-271

Kesavachandran CN, Haamann F, Nienhaus A (2013): Radiation exposure and adverse health effects of interventional cardiology staff. Rev Environ Contam Toxicol 222, 73-91

Kumari G, Kumar P, Wadhwa P, Aron M, Gupta NP, Dogra PN (2006): Radiation exposure to the patient and operating room personnel during percutaneous nephrolithotomy. Int Urol Nephrol 38, 207-210 
Leitlinie Urolithiasis s. S2k-Leitlinie zur Diagnostik, Therapie und Metaphylaxe der Urolithiasis 2015

Malmström T, Kröger RH (2006): Pupil shapes and lens optics in the eyes of terrestrial vertebrates. J Exp Biol 209, 18-25.

Mancini JG, Raymundo EM, Lipkin M, Zilberman D, Yong D, Bañez LL, Miller MJ, Preminger GM, Ferrandino MN. (2010): Factors affecting patient radiation exposure during percutaneous nephrolithotomy. J Urol 184, 2373-2377

Martin CJ (2009): A review of radiology staff doses and dose monitoring requirements. Radiat Prot Dosimetry $\underline{136}, 140-157$

Nakashima E, Neriishi K, Minamoto A (2006): A reanalysis of atomic-bomb cataract data, 2000-2002: a threshold analysis. Health Phys $\underline{90}, 154-160$

Nefzger MD, Miller RJ, Fujino T (1969): Eye findings in atomic bomb survivors of Hiroshima and Nagasaki: 1963-1964. Am J Epidemiol 89, 129-138

O'Connor U, Gallagher A, Malone L, O’Reilla G (2013): Occupational radiation dose to eyes from endoscopic retrograde cholangiopancreaticigraphy procedures in light of the revised eye lens dose limit from the International Commission on Radiological Protection. Br J Radiol 86(1022):20120289

Picano E, Vaňó E, Rehani MM, Cuoccolo A, Mont L, Bodi V, Bar O, Maccia C, Pierard L, Sicari R (2014): The appropriate and justified use of medical radiation in cardiovascular imaging: a position document of the ESC Associations of Cardiovascular Imaging, Percutaneous Cardiovascular Interventions and Electrophysiology. Eur Heart J $\underline{35}, 665-672$ 
Principi S, Delgado Soler C, Ginjaume M, Beltran Vilagrasa M, Rovira Escutia JJ, Duch MA (2015): Eye lens dose in interventional cardiology. Radiat Prot Dosimetry $\underline{165}, 289-293$

Ritter M, Krombach P, Martinschek A, Siegel FP, Schmitt M, Weicc C, Häcker A, Pelzer AE (2012): Radiation exposure during endourologic procedures using over-the-table flouroscopy sources. J Endourol 26, 47-51

S2k-Leitlinie zur Diagnostik, Therapie und Metaphylaxe der Urolithiasis 2015. Deutsche Gesellschaft für Urologie e.V. , 2015

Sánchez RM, Vano E, Fernández JM, Pifarré X, Ordiales JM, Rovira JJ, Carrera F, Goicolea J, Fernández-Ortiz A (2016): Occupational eye lens doses in interventional cardiology. A multicentric study. J Radiol Prot $\underline{36}, 133-143$

Sandilos P, Tsalafoutas I, Koutsokalis G, Karaiskos P, Georgiou E, Yakoumakis E, Vlahos L (2006): Radiation doses to patients from extracorporal shock wave lithotripsy. Health Phys $\underline{90}, 583-7$

Seo D, Kim KH, Kim JS, Han S, Park K, Kim J (2016): Evaluation of radiation doses in patient and medical staff during endoscopic retrograde cholangiopancreatography procedures. Radiat Prot Dosimetry $\underline{168}$, 516-522

Shannoun F, Blettner M, Schmidberger H, Zeeb H (2008): Strahlenschutz in der diagnostischen Radiologie. Dtsch Arztebl 105, 41-46

Thiessen G: Biologische Grundlagen der Strahlenwirkung. In: Hundeshagen H (Hrsg.): Radiologie. Springer, Heidelberg 1978, 28-71

Vano E, Fernandez JM, Resel LE, Moreno J, Sanchez RM (2016): Staff lens doses in interventional urology. A comparison with interventional radiology, cardiology and vascular surgery values. J Radiol Prot $\underline{36}, 37-48$ 
Vowinkel C: Intraoperative Strahlenbelastung der Patienten bei der ureterorenoskopischen Steinentfernung. Med. Diss. Göttingen 2011

Wolf N, Pendergrass W, Singh N, Swisshelm K, Schwartz J (2008): Radiation cataracts: mechanisms involved in their long delayed occurrence but then rapid progression. Mol Vis $\underline{14}, 274-285$

Worgul BV, Merriam JR Jr, Medvedovsky C (1989): Cortical cataract development - an expression of primary damage to the lens epithelium. Lens Eye Toxic Res $\underline{6}, 559-571$

Worgul BV, Kundiev Y, Likhtarev I, Sergienko N, Wegener A, Medvedovsky CP (1996): Use of subjective and nonsubjective methodologies to evaluate lens radiation damage in exposed populations--an overview. Radiat Environ Biophys $\underline{35}, 137-144$

Zagorska A, Romanova K, Hristova-Popova J, Vassileva J, Katzarov K (2015): Eye lens exposure to medical staff during endoscopic retrograde cholangiopancreatography. Phys Med 31, 781-784

Zintz C, Beebe DC (1986): Morphological and cell volume changes in the rat lens during the formation of radiation cataracts. Exp Eye Res $\underline{42}, 43-54$

Zöller G, Virsik-Köpp P, Vowinkel C (2013): Strahlenbelastung der Patienten bei der ureterorenoskopischen Steinentfernung. Urologe A $\underline{52}, 60-64$

Zöller G, Figel M, Denk J, Schulz K, Sabo A (2016): Strahlenbelastung der Augenlinse bei ureterorenoskopischen Eingriffen ohne und mit Strahlenschutzvisier. Urologe A $\underline{55}, 364-369$ 
Internetquellen:'

Bundesamt für Strahlenschutz:

http://www.asse.bund.de/DE/themen/ion/strahlenschutz/grenzwerte/grenzwerte.

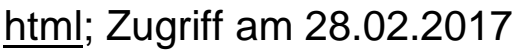

http://www.asse.bund.de/DE/themen/ion/wirkung/einfuehrung/einfuehrung.html, Zugriff am 28.02.2017

http://www.bfs.de/DE/themen/ion/umwelt/natuerliche-strahlenbelastung/ natuerliche-strahlenbelastung node.html, Zugriff am 28.02.2017 


\section{Danksagung}

Ich bedanke mich insbesondere bei meinem Doktorvater, Prof. Dr. med. Gerhard M. Zöller, der diese Arbeit angeregt und sehr engagiert betreut hat.

Vielen Dank auch Herrn Figel und Herrn Denk vom Helmholtz Zentrum München für die Bereitstellung und Auswertung der Augendosimeter und die Begleitung der Versuche sowie Dr. Reinert und Herrn Schulz aus der Klinik für Strahlentherapie des Klinikums Fulda für die leihweise Bereitstellung des Alderson-Phantoms.

Zudem danke ich ganz besonders meiner Freundin und ehemaligen Oberärztin Dr. Christiane Vowinkel und ihrem Mann, Ulrich Theis, sowie meinem Mann, Martin Dröge, für die Redigierung dieser Arbeit und die persönliche Unterstützung in dieser Zeit. 


\section{Lebenslauf}

Ich, Ana Sabo, wurde am 29.10.1981 als Tochter von Olivera und Ignac Sabo in Novi Sad (Serbien) geboren. Ich bin verheiratet und habe eine Tochter.

1988 wurde ich in Serbien eingeschult und besuchte zunächst die Sonja Marinkovic-Grundschule in Novi Sad. 1989 wechselte ich nach dem Umzug nach Deutschland in die Friedrich-von-Schiller-Grundschule in Wiesbaden und 1992 auf das Gutenberg-Gymnasium, ebenfalls in Wiesbaden. 1995 kehrte ich nach Serbien zurück; dort besuchte ich zunächst die Sonja Marinkovic-Schule und ab 1996 das Gymnasium Karlovačka Gimnazija in Sremski Karlovci. Nach einem erneuten Umzug nach Deutschland besuchte ich 1997 zunächst erneut das Gutenberg-Gymnasium in Wiesbaden und ab 1998 die GutenbergRealschule in Eltville. Nach dem Realschulabschluss 1999 wechselte ich an die Rheingau-Schule in Geisenheim. Dort legte ich 2002 mein Abitur ab.

2002 begann ich das Studium der Humanmedizin an der Johann Wolfgang Goethe-Universität Frankfurt, das ich 2008 mit dem Staatsexamen und der Note 1,5 beendete.

Meine Facharztausbildung begann ich am 01.01.2009 in der Klinik für Urologie und Kinderurologie des Klinikums Bad Hersfeld. Am 11. April 2014 erhielt ich nach bestandener Prüfung die Facharztanerkennung für Urologie.

Bis zum 31.10.2014 arbeitete ich zunächst weiter als Fachärztin am Klinikum Bad Hersfeld und wechselte dann an das Evangelische Klinikum Bethel Johannesstift in Bielefeld.

Seit dem 01.07.2016 arbeite ich, jetzt als Oberärztin, wieder in der Klinik für Urologie und Kinderurologie des Klinikums Bad Hersfeld. 